Research Article

\title{
Computational Analysis of Heat Transfer Intensification of Fractional Viscoelastic Hybrid Nanofluids
}

\author{
Mumtaz Khan (iD) and Amer Rasheed (iD \\ Department of Mathematics, School of Science and Engineering, Lahore University of Management Sciences, \\ Opposite Sector U, DHA, Lahore Cantt., Lahore 54792, Pakistan \\ Correspondence should be addressed to Amer Rasheed; amer.rasheed@lums.edu.pk
}

Received 3 May 2021; Accepted 10 September 2021; Published 19 October 2021

Academic Editor: Fahd Jarad

Copyright (C) 2021 Mumtaz Khan and Amer Rasheed. This is an open access article distributed under the Creative Commons Attribution License, which permits unrestricted use, distribution, and reproduction in any medium, provided the original work is properly cited.

\begin{abstract}
In the current article, we have performed computational analysis on convection heat transfer of a hybrid nanofluid in occurrences where porous media and the effect of magnetic force are involved. In order to assess the time-fractional derivatives, Caputo's notion is utilized while the Darcy-Forchheimer model is applied due to the involvement of the porous medium. Moreover, the boundary conditions are assumed to be nonuniform through the equilibrium between the surface tension and shear stress over a semi-infinite permeable flat surface. Keeping in view the complexity of the fractional derivative model and nonuniform boundary conditions, the problem in question is a complicated one. Accordingly, the coupled momentum and energy equation is linearized and the finite difference scheme is then applied and implemented in MATLAB Code R2020b. Furthermore, we have also offered a comprehensive analysis regarding error and convergence of the proposed numerical method. The newly introduced numerical technique to determine the numerical solutions and some unique and interesting deductions are established. From the computational results, one can conclude that the fluid motion in both hybrid and single nanofluids slows down due to magnetic field, porosity, and inertia coefficient as the magnetic and electric fields are synchronized due to the formation of the Lorentz force and viscous interference. We believe that our proposed numerical technique regarding employment of the fractional model for heat transfer application to the hybrid nanofluid over a semi-infinite nonuniform permeable surface along with variable heat flux is not found in the literature so far. Furthermore, the obtained results will be a valuable addition to fractional calculus from an engineering point of view.
\end{abstract}

\section{Introduction}

It has always remained one of the foremost objectives of researchers to formulate innovative methods to enhance the heat transfer rate in thermal systems, which will resultantly improve the productivity of heating systems while cutting off the expense simultaneously. Owing to a wide range of applications in various industries, convective heat transfer analysis inside a porous medium is one of the most appealing subjects. The employment of the porous medium in thermal systems results in two main advantages. First, the heat exchange rate is improved due to the enlargement of the heat transfer area. Second, the flow mixing is considerably increased owing to the chaotic movement of the fluid through appropriate pores. Accordingly, porous media have attracted a significant number of researchers to employ the same in heat transfer equipment in the recent past [1-5]. Thus, the characteristics of improved heat transfer can be referred to as facts from the literature. A porous medium is defined as a medium comprising a solid matrix with interconnected vacuums like metal foams and rocks. Figure 1 can be referred to have an illustration of carbonbased porous materials. Alibakhsh et al. [6], reviewed the nanofluid flow and heat transfer through porous media. Although the tendency of physical quantities in the scale of pores is not methodical, these are assessed in engineering applications in considerably large areas. Thus, a good number of researchers have analyzed various surfaces partially filled with the porous medium, and they 


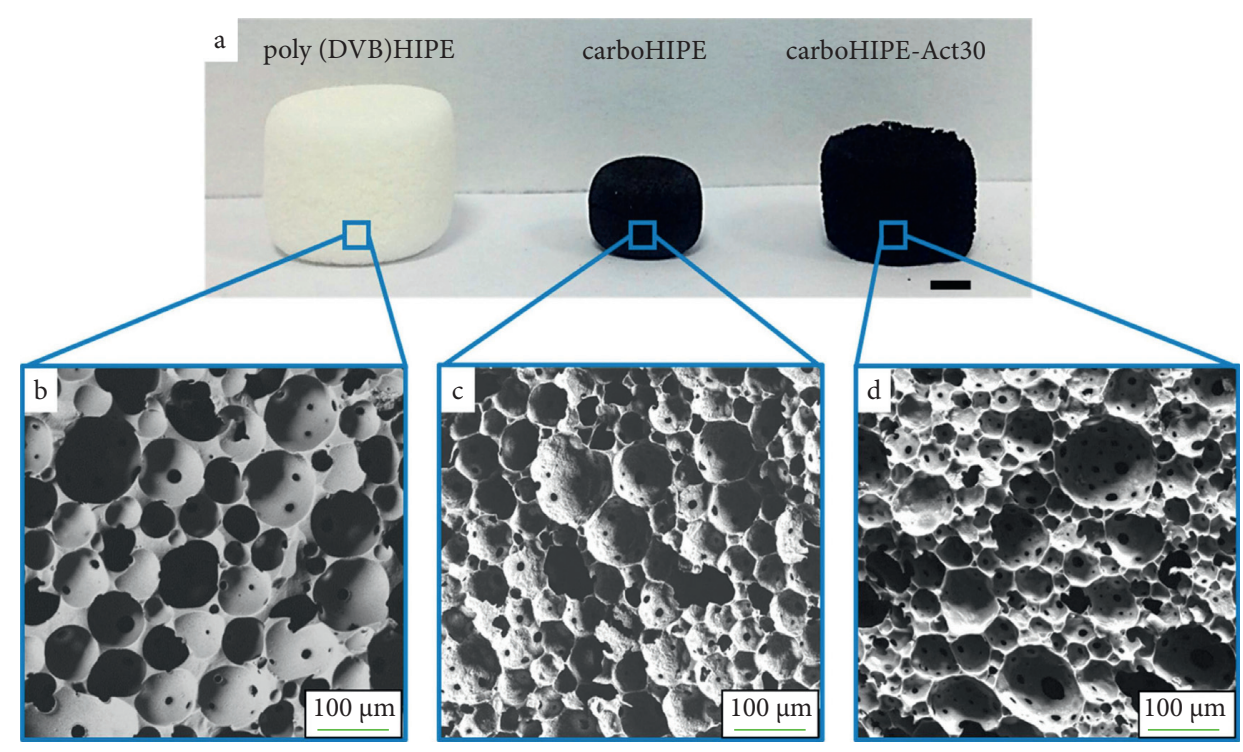

FIgURE 1: Few examples of permeable structures reliant on carbon [48].

established that maximum heat transfer could be obtained without filling the channel with the porous medium [2]. Without doubt, convection heat transfer in porous media is a well-known and imperative area of research. Nevertheless, porous media most often have excellent thermal efficiency. However, the weak thermal conductivity of fluids for heat transport causes severe limitations in enhancing heat transfer in cases where fluid flow is involved. From [7-9], it is evident that the addition of solid nanoparticles to conventional fluids results in a visible increase in thermal conductivity. Thus, instantaneous engagement of nanomaterials (i.e., suspensions of nanostructures) and permeable amalgamations may be referred as an effective technique to achieve substantial heat transfer. The scientific developments in the discipline of nanofluids lead to significant progress in various applications. Based on scientific research findings, nanofluids enhance the thermal conductivity and conductive heat transfer in relation to the base liquids.One cannot deny the fact that this modern kind of fluids received a remarkable acceptance from industry as these are deemed to be a good alternative of many traditional fluids, heat exchangers, and heat transport liquids [10] since nanofluids possess good temperature efficiency over liquids so that they can be utilized in numerous heating and ventilation applications (Figure 2(a)). The issue of water consumption in huge quantities and the production of excessive industrial waste can be addressed by increasing the heat transfer and simultaneously reducing water consumption.

Keeping in view the ultimate objective of enhancing convective heat transfer in different appliances, researchers have applied and tested multiple techniques like geometry modification, alteration of the boundary conditions, and enhancement of the thermophysical characteristics of the fluid in the recent past. The researchers and scientists have employed the technique of diffusing tiny solid particles in conventional fluids to maximize fluids' thermal characteristics. Previously, the particles with micrometer or millimeter measurements were used for this purpose. The use of these particles led to serious problems such as fouling, degradation, and loss of elevated pressure and, therefore, was not widely considered by researchers for future investigation. With the advancement in technology, nanotechnology was introduced, which further led to the discovery of nanoparticles which were of nanometer scale. Figure 3, can be referred for an illustration of nanoparticles. The modern type of dispersions named nanofluids is obtained by suspension of solid nanoparticles in ordinary liquids. These suspensions were named as "nanofluid" for the very first time in [11]. Choi presented a new type of fluid for heat transport containing tiny amounts of nanostructures that were metallic or nonmetallic. The particles described above were uniformly dispersed in a consistent manner and at a regular phase. The key advancements in nanofluid technology revealed the extraordinary capability of nanofluids in heat transfer applications, which provided a ground for research in the manufacturing area and academic research. The particles in nanocomposites vary from 1 to $100 \mathrm{~nm}$ on average. However, new research avenues, with simultaneous pros and cons, have been generated with the introduction of nanomaterials in fluid dynamics and heat transport. Investigators of nanomaterials need to have a thorough knowledge of the collective and rheological behaviors of nanofluids. This indicates that many researchers have made attempts to enhance the thermal performance utilizing the addition of various nanofluids to several heat exchangers (Figure 2(a)). Based on the base fluid and type of nanoparticles, nanofluids are divided into different categories. Quite a few researchers have also tried to investigate the thermal conductivity by adding metal nanomaterials to basic fluids like water $\left(\mathrm{H}_{2} \mathrm{O}\right)$, ethylene glycol (EG), and oil [12-15]. Nonmetals and nonmetal oxides are the nanoparticles that are also used to formulate different nanofluids $[16,17]$. Recently, the researchers have also paid attention to carbon nanotubes $[18,19]$. From [20-23], it is evident that 


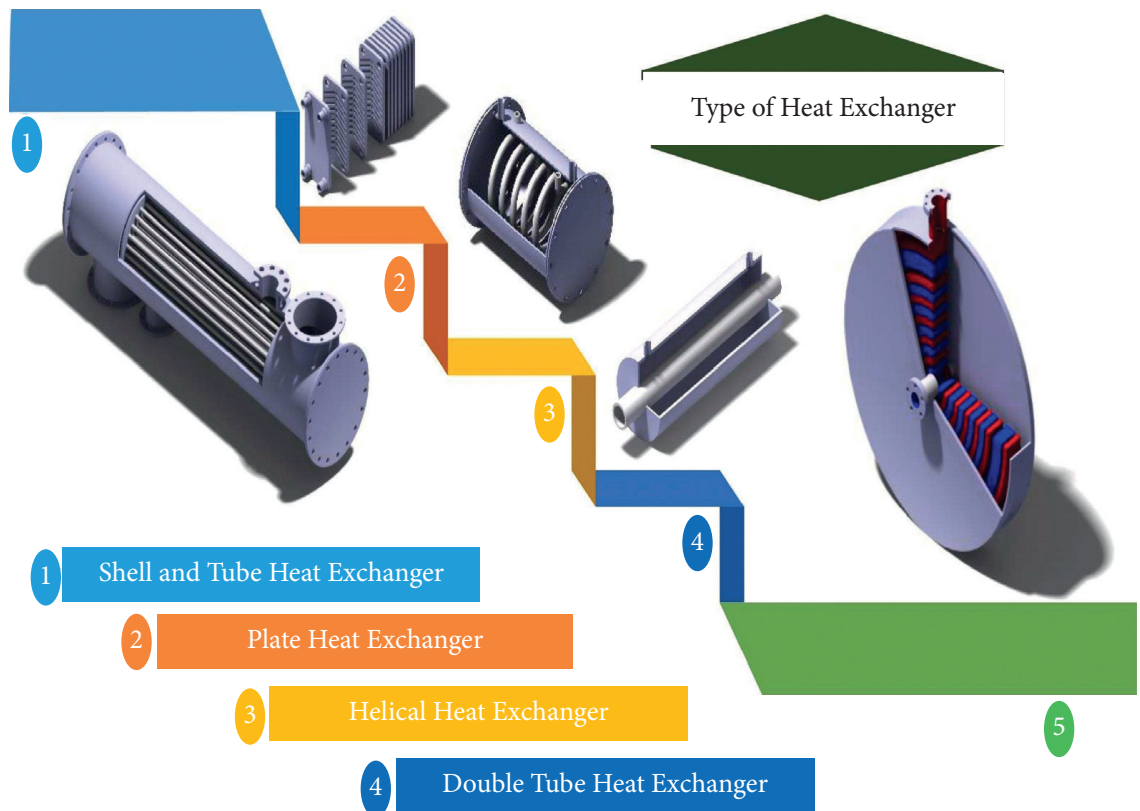

\section{Spiral Heat Excanger}

(a)

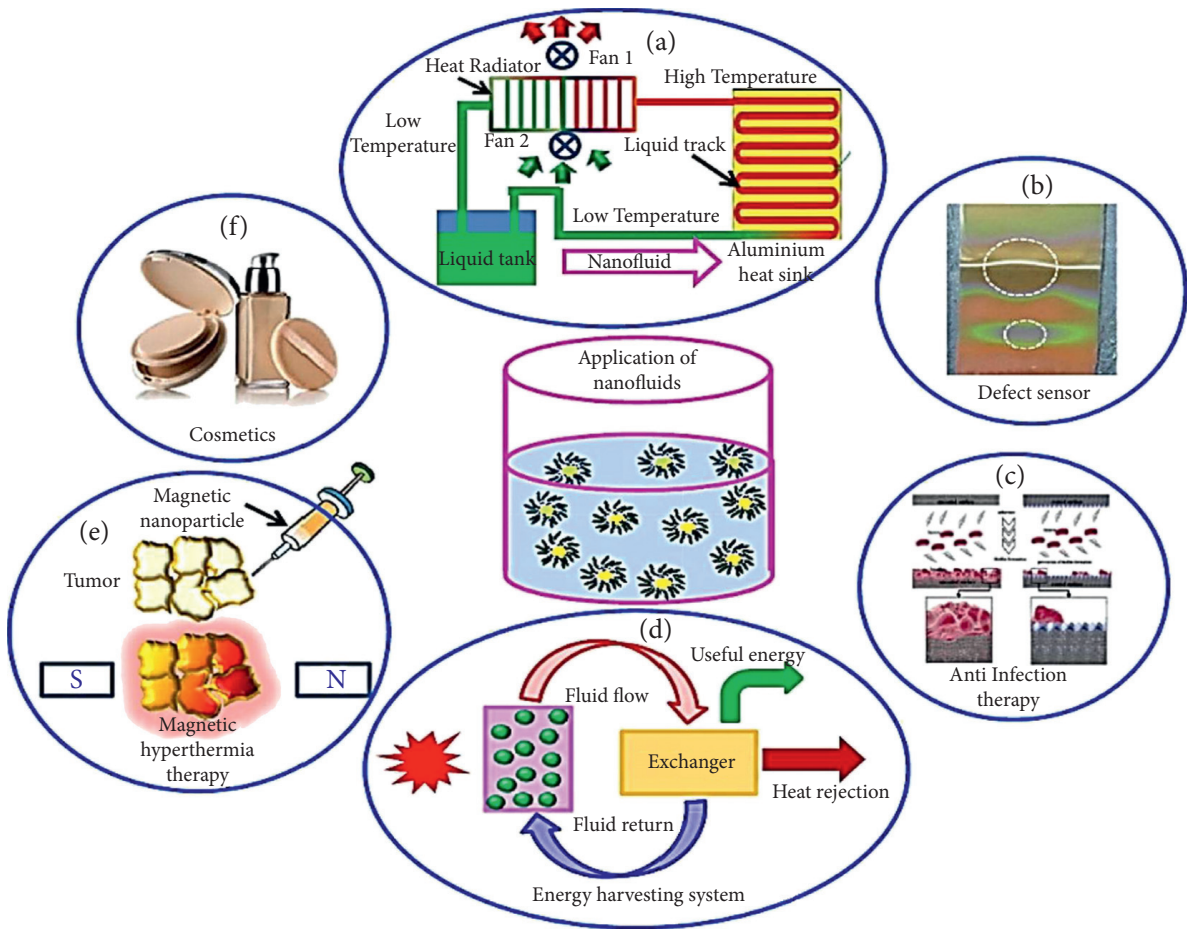

(b)

FIGURE 2: Common heat exchangers used in various industries [49] and primary applications of some nanofluids [50].

comprehensive studies addressing the experimental, numerical, and theoretical aspects are conducted by researchers to investigate the convective heat exchange of nanocomposites in various thermal environments. Figure 2(b) offers a pictorial view of nanofluid applications.

One can find several research articles wherein it is established that the existing models are not capable of predicting the thermal conductivity of nanofluids, and by the addition of nanoparticles in ordinary liquids, it can be enhanced multiple times. Here, Table 1 presents some published results regarding the thermal conductivity of nanofluids. Furthermore, some groups having aligned research goals made efforts to initiate organized research studies and entered into a modern age of nanoliquids, 


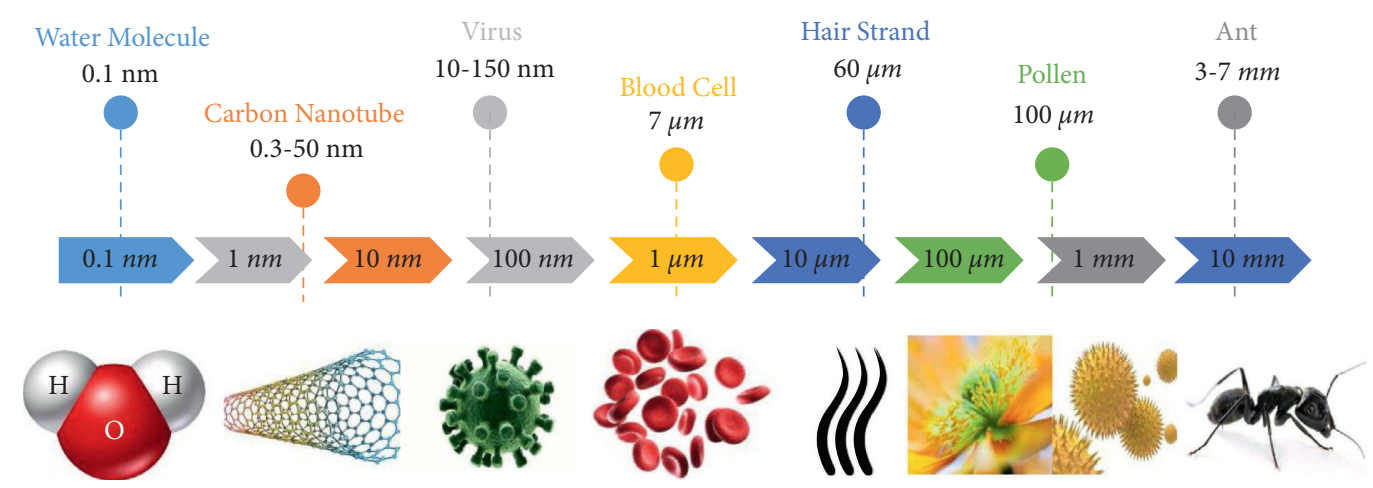

Figure 3: Comparison of different particle sizes [49].

Table 1: Studies on nanoliquid thermal conductivity [1].

\begin{tabular}{cccc}
\hline Refs & Nanofluids & NF concentrations & Improvement \\
\hline$[36]$ & $\mathrm{MWCNT}-\mathrm{SiO}_{2} / \mathrm{EG}$ & $0.86 \%$ & $20.1 \%$ \\
{$[37]$} & $\mathrm{ZnO}-\mathrm{EG}$ & $10.5 \mathrm{wt} \%$ & $13.0 \%$ \\
{$[38]$} & $\mathrm{MWCNT}$-water/EG & $1.0 \%$ & $34.7 \%$ \\
{$[39]$} & $\mathrm{Al}_{2} \mathrm{O}_{3}-\mathrm{Cu} /$ water & $1.0-5.0 \%$ & 10.0 to $13.56 \%$ \\
{$[25]$} & $\mathrm{MoSO}_{2}-\mathrm{SiO}_{2} /$ water & $10.0 \%$ & $0.8 \%$ \\
\hline
\end{tabular}

dealing with multiple kinds of nanomaterials at a time, termed as "hybrid nanofluids." The hybrid nanofluids can be considered the most modern kind of nanofluids where the main or base fluid contains more than one type of nanoparticles. More than one form of nanoparticles is utilized in such a category of nanocomposites. The type of nanofluids mentioned earlier possesses better properties when compared with mono nanofluids, and a number of researchers have paid attention to these fluids [24-26]. Values of the thermal conductivity of base fluids and different nanofluids are displayed in Figures 4(a) and 4 (b), respectively $[19,27-29]$. Very few research articles offer a comprehensive analysis of free convection characteristics of hybrid nanofluids in porous media. In the recent past, the study in [30] presented the numerical analysis of MHD hybrid nanofluids inside a T-shaped cavity. They studied the thermal enhancement of hybrid nanofluids by considering the mixture of MWCNT $-\mathrm{Fe}_{3} \mathrm{O}_{4}$. The viscosity of the nanofluid responds to the power of the magnetic force. The DarcyBrinkman-Forchheimer (DBF) integrative version was utilized to obtain the governing equations based on the dimensionless variables. Natural convection heat transfer owes a wide range of applications in several fields, including, but not limited to, renewable radiation, earth sciences, and development of thermal mass and nuclear power. In particular, free convection is substantial in instruments positioned at insufficient spaces, which is a common phenomenon in technological situations. In this case, heat transfer is caused by the fluid motion occurring due to buoyancy force and no external factor contributes towards heat transfer generation. Thus, the mechanisms involving free convection do not face the challenges of mechanical failures, which often occur in forced convective regimes. Moreover, free convection involves relatively low cost and produces immaterial turbulence from the shortage of established synchronous generators. Keeping in view the significance of natural convection in porous structures, several researchers have evaluated the impact of deployment of nanofluids in these media [31-33]. In the literature, a number of studies can be referred to that deal with the natural convection of nanomaterials in porous media with varying magnetic fields. For instance, magnetohydrodynamic (MHD) effects have attracted a number of scholars due to its widespread refrigerating capabilities of power plants and nuclear power plants, management of industrial waste, regulation of the spread of contaminants, etc. [34, 35].

The mathematical field that interacts with the study of defining real or complex order of the derivatives is called fractional calculus. In the recent past, significant developments have been witnessed in this area and this is being utilized in several engineering applications [40-44]. Researchers made several attempts to present a precise definition of the fractional derivatives. The definitions offered by Riemann-Liouville, Grunwald-Letnikov, and Caputo are the most widely accepted ones. The authors of [45] carried out a study to offer a comparative analysis of these definitions as follows:

Grunwald-Letnikov definition is

$$
\mathbf{G}_{\mathbf{L}} \mathbf{D}_{\mathbf{a}}^{\boldsymbol{\alpha}}=\lim _{h \longrightarrow 0} \sum_{j=0}^{N}\left(\begin{array}{c}
\alpha \\
j
\end{array}\right) f(t-j h) .
$$

Riemann-Liouville definition is

$$
\mathbf{R}_{\mathbf{L}} \mathbf{D}_{\mathbf{a}}^{\beta_{1}}=\frac{1}{\Gamma\left(m_{1}-\beta_{1}\right)} \frac{\mathrm{d}^{m_{1}}}{\mathrm{~d} \eta^{n}} \int_{a}^{t} f(t)(t-\eta)^{\beta_{1}-m_{1}+1} \mathrm{~d} \eta .
$$

Caputo definition is

$$
\mathbf{C}_{\mathbf{F}} \mathbf{D}_{\mathbf{a}}^{\beta_{1}}=\frac{1}{\Gamma\left(m_{1}-\beta_{1}\right)} \int_{a}^{t} f^{\left(m_{1}\right)}(t)(t-\eta)^{\beta_{1}-m_{1}+1} \mathrm{~d} \eta .
$$




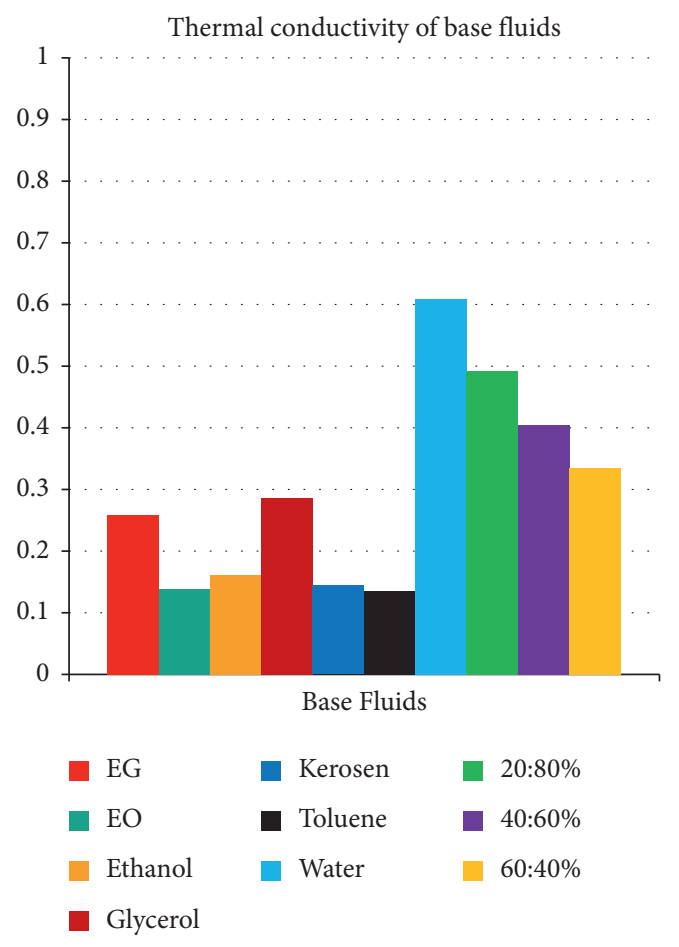

(a)

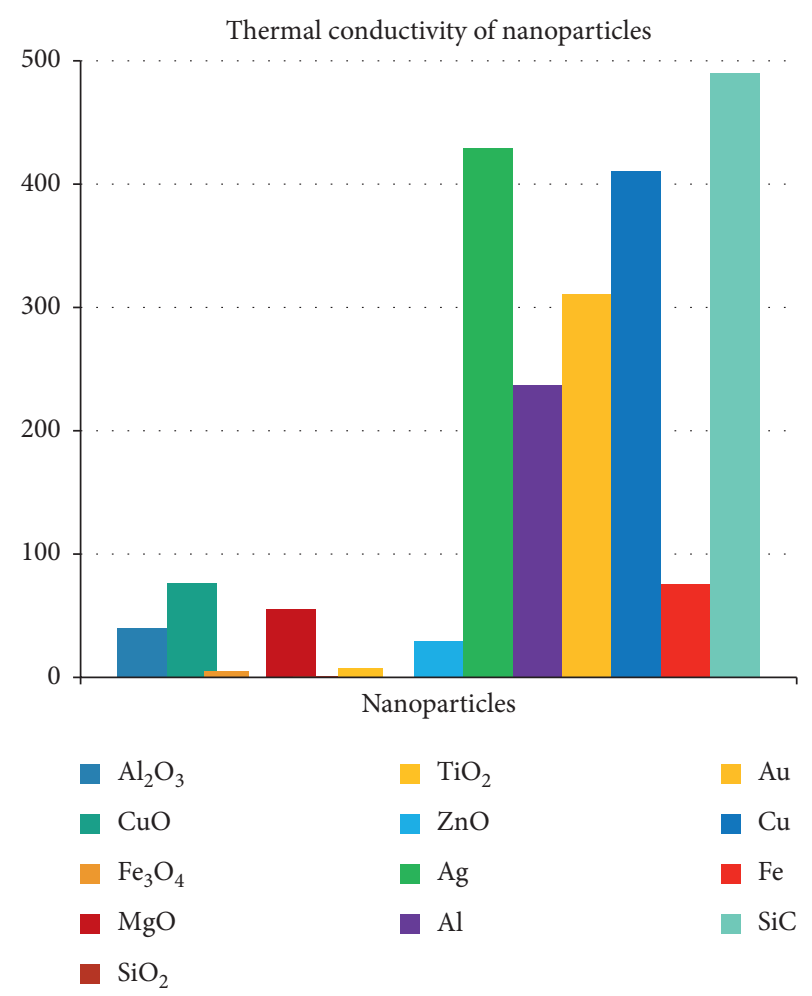

(b)

FIgURE 4: Thermal conductivity of different base fluids and nanofluids [49] (reprinted with permission from Elsevier).

In reference to the above, the literature offers attributes of Caputo and Reimann-Liouville derivatives, implementations of the time-fractional Navier-Stokes equations, and examination of many structural mathematical problems in the CFD (computational fluid dynamics) [46, 47].

The above analysis leads us to believe that the hybrid nanofluid has appeared as a promising heat transfer fluid despite having some unpredictability in the established outcomes and inadequate knowledge of the heat transfer tool in nanofluids. Researchers have extended research studies in the recent past by considering hybrid nanofluids, which are formed through suspension of unlike nanoparticles either in mixture or compound form. This provided us sufficient motivation to carry out the current study to present the fractional partial differential equations of the mixed convective flow over a nonuniform permeable semi-infinite surface filled with hybrid nanofluids subject to an applied magnetic field with variable heat flux. Here, the Darcy-Forchheimer model is utilized for the porous media, while Caputo's notion is used to approximate time-fractional derivatives. Moreover, two models, namely, fractional shear stress and Cattaneo heat flux, are presented in the constitutive relations with nonuniform boundary conditions. Thus, with mixed time-space derivatives in the convection terms, the nonlinear principal equations are formed. The finite difference method, together with $L_{1}$ algorithm is used to acquire the numerical solutions of the proposed problem. With the help of graphs and tables, it is shown how the parameters of interest affect velocity and temperature fields. Furthermore, the subject parameters are investigated in detail to affirm surface tension properties and heat transfer performance. In the current study, it is observed that the fractional-order derivative viscoelastic model gives more accurate and precise results than the integer-order viscoelastic model. Keeping in view the distinctive application like guided wave testing technique, one should have comprehensive knowledge of the subject model. Furthermore, it is pertinent to mention here that the uniform suction or injection is investigated widely by a number of researchers, but any researcher rarely studies nonuniform suction/injection over a semi-infinite flat surface filled with hybrid nanofluid. In industrial applications, the nonuniform suction/injection is more repeatedly observed than the uniform ones. Poor permeability is witnessed in a pipe-type filter system due to the accumulation of impurities at the inlet of the pipeline. In thermal protection applications where the wall has nonuniform slots, the skin friction is reduced by the adverse pressure gradient inside the boundary layer. The framework of the paper is given as follows: Section 2 deals with a comprehensive overview of the flow problem. Sections 3 and 4 refer to some significant notions related to engineering parameters and nondimensionless parameters. A finite difference method for the model equations has been developed in Section 5 and a precise numerical scheme is derived accordingly. A detailed discussion regarding the convergence of our proposed numerical scheme along with error analysis of the $L_{2}$ error norm is given in Section 6 . Section 7 is dedicated to candid discussion regarding results 
obtained through this study. In Section 8, a precise conclusion regarding the outcomes of the current study is given.

\section{Description of the Mathematical Problem}

Here in this article, we have taken into consideration the mixed convection flow of a generalized incompressible hybrid nanofluid over a dense porous leveled surface with flexible heat flux where the velocity of the even mainstream is taken as $u_{\infty}$. According to the boundary layer theory, the viscous effects have a leading role in the momentum equation, whereas pressure gradients have a negligible effect. The fluid is infused at the surface of the plate with uneven velocity $v_{w}(x)$ when the impurities in the fluids have partially blocked the holes. The fluid field outside the boundary layer zone is not altered as the magnitude of $v_{w}(x)$ is considerably small. Moreover, changes were observed in the power-law $q_{h}(x)=$ $A x^{n}$ and heat flux is generated and sustained at all time $t>0$. At the same time, free convection occurs due to temperature gradient and the uniform temperature of the atmosphere far from the surface is $T_{\infty}$. The initial condition is supposed to be fixed with ambient temperature $T_{\infty}$. A porous medium occupies the flow domain and the fluid employed is the $\mathrm{Al}_{2} \mathrm{O}_{3}-\mathrm{ZrO}_{2} / \mathrm{BF}$ hybrid nanofluid, for which Table 2 gives thermophysical characteristics. The Forchheimer model presents the porous matrix, and the local thermal equilibrium model between the mixture and the medium is maintained. A square velocity term representing the nonlinear drag impact that occurs because of a porous medium is introduced in Darcy law to obtain the Forchheimer model, the first-ever non-Darcy model. Furthermore, in a restricted temperature domain, the fluid characteristics are assumed to be constant. This model presents the momentary conduct of the heat transfer course of a stationary heat source. The fractional time derivatives $\alpha$ and $\gamma$ contribute to controlling fluid flow and heat propagation. All other physical parameters are constant, and viscous dissipation in the energy equation is almost insignificant. We have utilized the fractional Caputo time derivative to develop a mathematical model [51]. Assuming all the above conditions, the principal boundary layer equations of momentum and energy for the fractional hybrid nanofluid are as follows [25, 52-54]:

$$
\begin{gathered}
\frac{\partial u}{\partial x}+\frac{\partial v}{\partial y}=0 \\
\left(\frac{\partial u}{\partial t}+u \frac{\partial u}{\partial x}+v \frac{\partial u}{\partial y}\right)=\frac{1}{\rho_{h n f}} \frac{\partial \sigma_{x y}}{\partial y}+g \beta_{h n f}\left(T-T_{\infty}\right),
\end{gathered}
$$

TABLE 2: Thermophysical properties of fluid and nanoparticles [52, 55-58].

\begin{tabular}{lccc}
\hline $\begin{array}{l}\text { Physical } \\
\text { property }\end{array}$ & $\begin{array}{c}\text { Base fluid } \\
(\mathrm{CTAC} / \mathrm{NaSal} \text {-water })\end{array}$ & $\mathrm{Al}_{2} \mathrm{O}_{3}$ & $\mathrm{ZrO}_{2}$ \\
\hline$\rho /\left(\mathrm{kg} \cdot \mathrm{m}^{-3}\right)$ & 997.1 & 3970 & 5600 \\
$c_{p} /(\mathrm{J} \cdot \mathrm{kg} \cdot \mathrm{K})$ & 4179 & 765 & 481 \\
$k /(\mathrm{W} \cdot \mathrm{mk})$ & 0.613 & 40 & 2.8 \\
$\beta /\left(\mathrm{K}^{-1}\right)$ & $210 \times 10^{-6}$ & $38.87 \times 10^{-6}$ & $5.57 \times 10^{-6}$ \\
$\sigma /(\mathrm{S} \cdot \mathrm{m})$ & 0.05 & $3.67 \times 10^{7}$ & $6 \times 10^{2}$ \\
\hline
\end{tabular}

$$
\left(\frac{\partial T}{\partial t}+u \frac{\partial T}{\partial x}+v \frac{\partial T}{\partial y}\right)=-\frac{1}{\left(\rho c_{p}\right)_{h n f}} \frac{\partial q}{\partial y},
$$

where $u$ and $v$ represent the flow velocity, $T$ and $\rho_{\text {hnf }}$ denote the hybrid fluid density and fluid temperature, respectively, $\left(c_{p}\right)_{h n f}$ is the hybrid nanofluid heat capacity at constant pressure, $g$ is the acceleration, and $\beta_{h n f}$ is the thermal expansion coefficient of hybrid nanofluid, and $\sigma_{x y}$ and $q$ are the shear stress and heat flux.

In Table 3, $\phi_{1}$ and $\phi_{2}$, respectively, denote the volume fractions of $\mathrm{Al}_{2} \mathrm{O}_{3}$ and $\mathrm{ZrO}_{2}$ nanomaterials, where $\phi_{1}=\phi_{2}=$ 0 is the normal fluid, $\mu$ is the dynamic viscosity, $\rho$ is the standard liquid, $\mu$ reflects the elastic viscosity, $\rho$ is the density, $c_{p}$ is the specific heat at constant strain, $\rho c_{p}$ is the heat capacity, $k$ is the thermal conductivity, and $\sigma$ is the electrical conductivity in which $h n f, n f, f$, and $s 1$ and $s 2$ subscripts represent hybrid nanofluid, nanofluid, fluid, and solid nanoparticles for $\mathrm{Al}_{2} \mathrm{O}_{3}$ and $\mathrm{ZrO}_{2}$, respectively.

In the momentum equation, the constitutive relation of the nanoliquid with the Caputo fractional model is incorporated as

$$
\sigma_{x y}+\lambda_{1}^{\alpha} \frac{\partial^{\alpha} \sigma_{x y}}{\partial t^{\alpha}}=\mu_{h n f} \frac{\partial u}{\partial y},
$$

where $\lambda_{1}, \mu_{h n f}$, and $\alpha$ are the relaxation time for momentum equation, hybrid nanofluid viscosity, and memory term for velocity, respectively. For the heat transfer enhancement, we have added the Darcy-Forchheimer porosity medium to the momentum equation. The strain of Darcy-Forchheimer model to the liquid that passes via the porous medium is described as [60]

$$
\text { Darcy }- \text { Forchheimer }=-\frac{\psi}{k}[u]-\frac{C_{F} \psi}{\sqrt{k}}\left[u^{2}\right] .
$$

The permeability and porosity of the porous medium are $k$ and $\psi$, respectively.

Combining equations (5), (7), and (8), the momentum equation with mixed derivatives of time and space is defined as

$$
\left\{\begin{array}{l}
\left(1+\lambda_{1 \mathbf{C}_{\mathrm{F}}}^{\alpha} \mathbf{D}_{\mathbf{t}}^{\alpha}\right)\left(\frac{\partial u}{\partial t}+u \frac{\partial u}{\partial x}+v \frac{\partial u}{\partial y}\right)=v_{h n f} \frac{\partial^{2} u}{\partial y^{2}}-\frac{\psi v_{h n f}}{k}\left(1+\lambda_{1 \mathbf{C}_{\mathrm{F}}}^{\alpha} \mathbf{D}_{\mathbf{t}}^{\alpha}\right) u-\frac{C_{F} \phi}{\sqrt{k}}\left(1+\lambda_{1 \mathbf{C}_{\mathrm{F}}}^{\alpha} \mathbf{D}_{\mathbf{t}}^{\alpha}\right) u^{2} \\
-\frac{\sigma_{h n f} B_{0}^{2}}{\rho_{h n f}}\left(1+\lambda_{1 \mathrm{C}_{\mathrm{F}}}^{\alpha} \mathbf{D}_{\mathbf{t}}^{\alpha}\right) u+g \beta_{h n f}\left(1+\lambda_{1 \mathrm{C}_{\mathrm{F}}}^{\alpha} \mathbf{D}_{\mathbf{t}}^{\alpha}\right)\left(T-T_{\infty}\right),
\end{array}\right.
$$




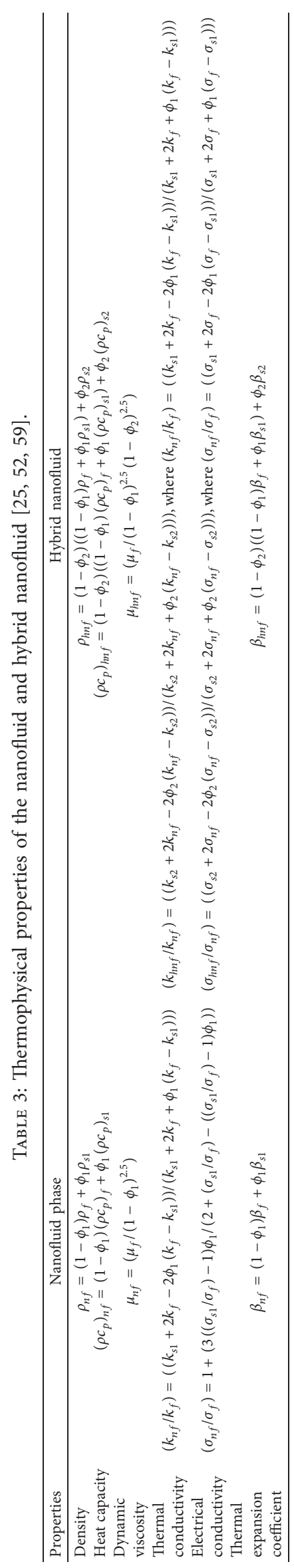


utilizing the latest Taylor series of fractional order, which establishes thermoelasticity with time-delay and memorydependent derivative; the fractional Cattaneo heat conduction model is [61]

$$
q+\lambda_{2}^{\gamma} \frac{\partial^{\gamma} q}{\partial t^{\gamma}}=K_{h n f} \nabla T
$$

where $\lambda_{2}$ and $\gamma$ are relaxation time for energy equation and the control parameter for the same equation; this model removes the dilemma of the infinite speed of energy distribution in thermoelectric liquid and increases the performance of the thermoelectric substance as per the specification of permissible fractional derivative order values $\gamma$. Analogously, the fractional temperature equation can be derived by incorporating equation (10) into (6) as

$$
\left(1+\lambda_{2 \mathbf{C}_{\mathrm{F}}}^{\gamma} \mathbf{D}_{\mathbf{t}}^{\gamma}\right)\left(\frac{\partial T}{\partial t}+u \frac{\partial T}{\partial x}+v \frac{\partial T}{\partial y}\right)=\frac{K_{h n f}}{\left(\rho c_{p}\right)_{h n f}} \frac{\partial^{2} T}{\partial y^{2}} .
$$

For the above-considered equations, the required initial and boundary conditions are stated as follows:

$$
\left\{\begin{array}{l}
u(x, 0, t):=0, \\
v(x, 0, t):=v_{w} \\
K\left(\frac{\partial T(x, 0, t)}{\partial y}\right)=-q_{w}(x), \quad t \geq 0 x \geq 0, \\
u(x,+\infty, t):=u_{\infty}, \\
v(x,+\infty, t):=0, \\
T(x,+\infty, t) \longrightarrow T_{\infty}, \quad t \geq 0, x \geq 0, \\
u(0, y, t):=u_{\infty}, \\
v(0, y, t):=0, \\
T(0, y, t)=T_{\infty}, \quad t \geq 0 y>0, \\
\partial_{y} u(x, y, 0)=0, \\
T(x, y, 0)=0=\partial_{y} v(x, y, 0), \\
u(x, 0)=0=\partial_{y} T(x, y, 0), \quad t \geq 0 y>0
\end{array}\right.
$$

\section{Significant Parameters of Practical Interest}

The drag coefficient and heat transfer rate are the important variables for the above models, which can be considered the measurement of the surface shear stress and heat transport ability of the convection flow system simultaneously. From the constitutive fractional equations (7) and (10), the local skin friction coefficient and the Nusselt number can be interpreted as follows:

$$
\begin{aligned}
& \left(1+\lambda_{1 \mathbf{C}_{\mathbf{F}}}^{\alpha} \mathbf{D}_{\mathbf{t}}^{\alpha}\right) C_{f}:=-\operatorname{Re} A_{1}\left(\frac{\partial u}{\partial y}\right)_{y=0}, \\
& \left(1+\lambda_{2 \mathbf{C}_{\mathbf{F}}}^{\gamma} \mathbf{D}_{\mathbf{t}}^{\gamma}\right) \mathrm{Nu}=-L A_{4}\left(\frac{\partial T}{\partial y}\right)_{y=0} .
\end{aligned}
$$




\section{Nondimensional Caputo Fractional Flow Equations}

To nondimensionalize the above equations, the corresponding dimension variables are added to

$$
\begin{aligned}
\widehat{x^{*}} & :=x L^{-1}, \\
\widehat{y^{*}} & :=y L^{-1}, \\
\widehat{u^{*}} & :=u u_{\infty}^{-1}, \\
\widehat{v^{*}} & :=v u_{\infty}^{-1}, \\
\widehat{t^{*}} & :=\left(t u_{\infty}\right) L^{-1}, \\
\widehat{T^{*}} & :=\frac{\left(T-T_{\infty}\right) K}{L q_{w}(L)},
\end{aligned}
$$

where $L$ is the characteristic length. Governing equations (4), (9), and (11) are (for simplicity, the dimensionless mark $\widehat{*}$ is omitted):

$$
\left\{\begin{array}{l}
\frac{\partial u}{\partial x}+\frac{\partial v}{\partial y}=0, \\
\left(1+\lambda_{1 \mathbf{C}_{\mathrm{F}}}^{\alpha} \mathbf{D}_{\mathbf{t}}^{\alpha}\right)\left(\frac{\partial u}{\partial t}\right)+\left(1+\lambda_{1 \mathbf{C}_{\mathrm{F}}^{\alpha}}^{\alpha} \mathbf{D}_{\mathbf{t}}^{\alpha}\right)\left(u \frac{\partial u}{\partial x}\right) \\
+\left(1+\lambda_{1 \mathbf{C}_{\mathrm{F}}}^{\alpha} \mathbf{D}_{\mathbf{t}}^{\alpha}\right)\left(v \frac{\partial u}{\partial y}\right)=\left(\frac{A_{1} A_{2}}{\operatorname{Re}}\right) \frac{\partial^{2} u}{\partial y^{2}} \\
-\frac{1}{\operatorname{Re}}\left(\lambda A_{1} A_{2}+\operatorname{Ha}\left(\frac{A_{3}}{A_{2}}\right)\right)\left(1+\lambda_{1 \mathbf{C}_{\mathrm{F}}}^{\alpha} \mathbf{D}_{\mathbf{t}}^{\alpha}\right) u \\
-\frac{F_{r}}{\operatorname{Re}}\left(1+\lambda_{1 \mathbf{C}_{\mathrm{F}}}^{\alpha} \mathbf{D}_{\mathbf{t}}^{\alpha}\right) u^{2}+\frac{\Lambda_{1}}{\operatorname{Re}}\left(\frac{\beta_{h n f}}{\beta_{B F}}\right)\left(1+\lambda_{1 \mathbf{C}_{\mathrm{F}}}^{\alpha} \mathbf{D}_{\mathbf{t}}^{\alpha}\right) T \\
\left(1+\lambda_{2 \mathbf{C}_{\mathrm{F}}}^{\gamma} \mathbf{D}_{\mathbf{t}}^{\gamma}\right)\left(\frac{\partial T}{\partial t}\right)+\left(1+\lambda_{2 \mathbf{C}_{\mathrm{F}}}^{\gamma} \mathbf{D}_{\mathbf{t}}^{\gamma}\right)\left(u \frac{\partial T}{\partial x}\right) \\
+\left(1+\lambda_{2 \mathbf{C}_{\mathrm{F}}}^{\gamma} \mathbf{D}_{\mathbf{t}}^{\gamma}\right)\left(v \frac{\partial T}{\partial y}\right)=\frac{1}{\operatorname{RePr}}\left(\frac{A_{4}}{A_{5}}\right) \frac{\partial^{2} T}{\partial y^{2}}
\end{array}\right.
$$

together with the following flow conditions:

$$
\left\{\begin{array}{l}
u(x, 0, t):=0, \\
v(x, 0, t):=v_{w}, \\
\left(\frac{\partial T(x, 0, t)}{\partial y}\right)=-x^{n}, \quad t \geq 0, x \geq 0, \\
u(x,+\infty, t):=1, \\
v(x,+\infty, t):=0, \\
T(x,+\infty, t) \longrightarrow 0, \quad t \geq 0, x \geq 0, \\
u(0, y, t):=1, \\
v(0, y, t):=0, \\
\partial_{y} u(x, y, 0)=0, \\
v(x, y, t)=0, \quad t \geq 0, y>0, \\
T(x, y, 0)=0=\partial_{y} T(x, y, 0), \quad t \geq 0, y>0,
\end{array}\right.
$$

where 


$$
\begin{aligned}
& \int \frac{\mu_{h n f}}{\mu_{B F}}=(1-\phi)^{-2.5} \\
& \phi=\phi_{\mathrm{Al}_{2} \mathrm{O}_{3}}+\phi_{\mathrm{ZrO}_{2}}=A_{1} \text {, } \\
& \frac{\rho_{\text {hnf }}}{\rho_{B F}}=(1-\phi)+\frac{\phi_{\mathrm{Al}_{2} \mathrm{O}_{3}} \rho_{\mathrm{Al}_{2} \mathrm{O}_{3}}}{\rho_{B F}}+\frac{\phi_{\mathrm{ZrO}_{2}} \rho_{\mathrm{ZrO}_{2}}}{\rho_{B F}}=A_{2}, \\
& \lambda=\frac{\psi L^{2}}{k}, \\
& \frac{\sigma_{h n f}}{\sigma_{B F}}=\left[1+\frac{3 \phi_{\mathrm{Al}_{2} \mathrm{O}_{3}} \sigma_{\mathrm{Al}_{2} \mathrm{O}_{3}}+\phi_{\mathrm{ZrO}_{2}} \sigma_{\mathrm{ZrO}_{2}}+3 \phi \sigma_{B F}}{\sigma_{\mathrm{Al}_{2} \mathrm{O}_{3}}\left(1-\phi_{\mathrm{Al}_{2} \mathrm{O}_{3}}\right)+\sigma_{\mathrm{ZrO}_{2}}\left(1-\phi_{\mathrm{ZrO}_{2}}\right)+(2+\phi) \sigma_{f}}\right]=A_{3} \text {, } \\
& \operatorname{Pr}:=\frac{\nu\left(\rho c_{p}\right)_{B F}}{K_{B F}} \\
& F_{r}=\frac{C_{F} \psi L^{2} u_{\infty}}{\sqrt{k} v} \\
& \Lambda_{1}=\frac{\mathrm{Gr}}{\mathrm{Re}} \\
& \frac{\beta_{h n f}}{\beta_{B F}}=(1-\phi)+\frac{\phi_{\mathrm{Al}_{2} \mathrm{O}_{3}} \beta_{\mathrm{Al}_{2} \mathrm{O}_{3}}}{\beta_{B F}}+\frac{\phi_{\mathrm{ZrO}_{2}} \beta_{\mathrm{ZrO}_{2}}}{\beta_{B F}}, \\
& A_{6}=\frac{A_{3}}{A_{2}}, \\
& A_{7}=\frac{A_{4}}{A_{5}}, \\
& \frac{k_{h n f}}{k_{B F}}=\frac{2(1-\phi) k_{B F}+\left(1+2 \phi_{\mathrm{Al}_{2} \mathrm{O}_{3}}\right) k_{\mathrm{Al}_{2} \mathrm{O}_{3}}+\left(1+2 \phi_{\mathrm{ZrO}_{2}}\right) k_{\mathrm{ZrO}_{2}}}{(2+\phi) k_{B F}+\left(1-\phi_{1}\right) k_{\mathrm{Al}_{2} \mathrm{O}_{3}}+\left(1-\phi_{2}\right) k_{\mathrm{ZrO}_{2}}}=A_{4} \text {, } \\
& \mathrm{Gr}=\frac{g \beta_{B F} L^{4} q_{w}(L)}{K v^{2}} \\
& \frac{\left(\rho c_{p}\right)_{h n f}}{\left(\rho c_{p}\right)_{B F}}=(1-\phi)+\frac{\phi_{\mathrm{Al}_{2} \mathrm{O}_{3}}\left(\rho c_{p}\right)_{\mathrm{Al}_{2} \mathrm{O}_{3}}+\phi_{\mathrm{ZrO}_{2}}\left(\rho c_{p}\right)_{\mathrm{ZrO}_{2}}}{\left(\rho c_{p}\right)_{B F}}=A_{5}, \\
& \mathrm{Ha}=\frac{\sigma_{B F} L^{2} B_{0}^{2}}{\mu_{B F}}
\end{aligned}
$$


The local drag factor and heat transfer rate in steady state are achieved by solving (13) at each time step, as $t_{k}$ approaches the convergence time:

$$
\begin{aligned}
& C_{f}=\left[r_{1} \sum_{s=1}^{k-1}\left(\alpha_{s-1}-\alpha_{s} C_{f}\left(t_{k-s}\right)\right)-\operatorname{Re} A_{1} \frac{(\partial u / \partial y)_{y=0}}{1+r_{1}}\right], \\
& \mathrm{Nu}=\left[r_{3} \sum_{q=1}^{k-1}\left(\gamma_{q-1}-\gamma_{q} \mathrm{Nu}\left(t_{k-q}\right)\right)-L A_{4} \frac{(\partial T / \partial y)_{y=0}}{1+r_{3}}\right] .
\end{aligned}
$$

Thus, the average drag coefficient and heat transfer rate satisfy, respectively,

$$
\begin{aligned}
\left(1+\lambda_{1 \mathbf{C}_{\mathbf{F}}}^{\alpha} \mathbf{D}_{\mathbf{t}}^{\alpha}\right) \overline{C_{f}} & :=\frac{A_{1}}{\operatorname{Re}} \int_{0}^{1}\left(\frac{\partial u}{\partial y}\right)_{y=0},\left(1+\lambda_{2 \mathbf{C}_{\mathbf{F}}}^{\gamma} \mathbf{D}_{\mathbf{t}}^{\gamma}\right) \mathrm{d} x \\
\overline{\mathrm{Nu}} & =-A_{4} \int_{0}^{1}\left(\frac{\partial T}{\partial y}\right)_{y=0}, \\
C_{f} & \left.=\left[r_{1} \sum_{s=1}^{k-1}\left(\alpha_{s-1}-\alpha_{s}\right) \overline{C_{f}}\left(t_{k-s}\right)\right)-\frac{A_{1}}{\operatorname{Re}} \int_{0}^{1} \frac{(\partial u / \partial y)_{y=0}}{1+r_{1}}\right], \\
\mathrm{Nu} & \left.=\left[r_{3} \sum_{q=1}^{k-1}\left(\gamma_{q-1}-\gamma_{q}\right) \overline{\mathrm{Nu}}\left(t_{k-q}\right)\right)-A_{4} \int_{0}^{1} \frac{(\partial T / \partial y)_{y=0}}{1+r_{3}}\right] .
\end{aligned}
$$

The initial conditions provide us with $u, v$, and $T$ values at time $t=0$ in the prescribed domain. Throughout a single time step, the preceding time-level variables, which are present in coefficients or sums, are assumed as constants. The values of $u, v$, and $T$ at a specific time level $k$ are determined at each internal nodal point on a particular $i$-level. The right side of the linear equations contains the $i$-level at the edge of the surface, and the initial point is located. In addition, the $T$ values are calculated for each inner grid point on a particular $i$ at the $k$-th time interval. Moreover, the values of $u$ at the $k$-th time level are obtained by using the already determined values of $T$ at the $k$-th time level along with $T$ and $u$ values in the preceding time levels drawing back to the initial time. Thus, at each internal nodal point on a specific $i$-level, the values of $v$ are determined. The process, as mentioned earlier, of determining values of different parameters is reiterated till the selected boundary is attained. Consequently, the values of $u, v$, and $T$ are determined at all nodal points in the required domain. Furthermore, the values of $C_{f}, \mathrm{Nu}, \overline{C_{f}}$, and $\overline{\mathrm{Nu}}$ are obtained along similar lines, where the trapezoidal compound rule is utilized to calculate the integrals. The time process is reiterated till the steady state is attained when the differences observed are less than $10^{-5}$ at all grid cells among the values of velocity $u$ and temperature $T$ at two sequential time levels. The computing region is considered to be a rectangle with variables $L_{x}=1$ and $L_{y}=5$. A particular point $L_{x}$ on the surface can be theoretically extended to further distances. Against each $y \longrightarrow T, L_{y}$ which is located outside the momentum and energy boundary layers.

\section{Numerical Technique}

This section deals with the numerical solution of fractional diffusion equations. In order to determine the numerical solution of nonlinear equations (15) and (16) mixed with space and time derivatives, the earlier formulated finite difference method together with the $L_{1}$ algorithm is used. The $L_{1}$ scheme developed in [62] is utilized to solve Caputo fractional derivative and is considered one of the most valuable and effective methods for discretizing one-dimensional space-time derivative.

5.1. Discretization Method. The unsteady coupled system of nonlinear fractional time and space derivative equations (15) and (16) are solved by a well-known finite difference process (FDM) along with $L_{1}$ in nonuniform boundary condition (16). We have $x_{i}=i \delta x$, where $i=0,1,2,3, \ldots, M ; y_{j}=j \delta y$, where $j=0,1,2,3, \ldots, N$; and $t_{k}=k \tau$, where $k=0,1,2, \ldots, R$, in which $\delta x=L_{x} / M$ and $\delta y=L_{y} / N$ are space steps and $\tau$ is the time step. The numerical solution of velocity and temperature at the mesh point $\left(x_{i}, y_{j}, t_{k}\right)$ is denoted as $u_{i j}^{k}$ and $T_{i j}^{k}$. To discretize the time-fractional derivative, the $L_{1}$ approach is applied to $(0<\alpha<1)$ and $(0<\gamma<1)$ as [62] 


$$
\left\{\begin{aligned}
D_{t}^{\alpha} u\left(t_{k}\right) & :=\frac{\tau^{-\alpha}}{\Gamma(2-\alpha)} \sum_{s=0}^{k-1} \alpha_{s}\left[u\left(t_{k-s}\right)-u\left(t_{k-s-1}\right)\right]+O\left(\tau^{2-\alpha}\right) \\
& =\frac{\tau^{-\alpha}}{\Gamma(2-\alpha)}\left[\alpha_{0} u\left(t_{k}\right)-\alpha_{k-1} u\left(t_{0}\right)-\sum_{s=1}^{k-1}\left(\alpha_{s-1}-\alpha_{s}\right) u\left(t_{k-s}\right)\right]+O\left(\tau^{2-\alpha}\right),
\end{aligned}\right.
$$

where $\alpha_{s}=(s+1)^{1-\alpha}-(s)^{1-\alpha}$, in which $s=0,1,2, \ldots, r$.

By the backward difference, the time derivative is discretized:

$$
\frac{\partial u}{\partial t}=\frac{u_{i, j}^{k}-u_{i, j}^{k-1}}{\tau}+O(\tau) .
$$

By linearization processing, the implicit difference scheme is considered for the space derivatives:

$$
\begin{aligned}
& u \frac{\partial u}{\partial x}=u_{i, j}^{k-1} \frac{u_{i, j}^{k}-u_{i, j}^{k-1}}{\delta x}+O(\delta x), \\
& v \frac{\partial u}{\partial x}=v_{i, j}^{k-1} \frac{u_{i, j}^{k}-u_{i, j}^{k-1}}{\delta y}+O(\delta y) .
\end{aligned}
$$

The finite difference approximations of equation (15) using Crank-Nicolson method is defined below :

$$
\frac{\partial^{2} u}{\partial y^{2}}=\frac{u_{i, j+1}^{k}-2 u_{i, j}^{k}+u_{i, j-1}^{k}+u_{i, j+1}^{k-1}-2 u_{i, j}^{k-1}+u_{i, j-1}^{k-1}}{2(\delta y)^{2}}+O\left(\delta y^{2}\right) .
$$

Then, equations (22) and (23) are substituted into the fractional derivatives:

$$
\left\{\begin{array}{l}
\frac{\partial^{\alpha+1} u}{\partial t^{\alpha+1}}=\frac{\tau^{-1-\alpha}}{\Gamma(2-\alpha)}\left[u_{i j}^{k}-u_{i j}^{k-1}-\sum_{s=1}^{k-1}\left(\alpha_{s-1}-\alpha_{s}\right)\left(u_{i j}^{k-s}-u_{i j}^{k-s-1}\right)\right], \\
\frac{\partial^{\alpha}}{\partial t^{\alpha}}\left(u \frac{\partial u}{\partial x}\right)=\frac{\tau^{-\alpha}}{\delta x \Gamma(2-\alpha)}\left[u_{i j}^{k-1}\left(u_{i j}^{k}-u_{i-1 j}^{k}\right)-\sum_{s=1}^{k-1}\left(\alpha_{s-1}-\alpha_{s}\right) u_{i j}^{k-s-1}\left(u_{i j}^{k-s}-u_{i-1 j}^{k-s}\right)\right]+O\left(\tau^{2-\alpha}+\delta x\right), \\
\frac{\partial^{\alpha}}{\partial t^{\alpha}}\left(v \frac{\partial u\left(t_{k}\right)}{\partial y}\right)=\frac{\tau^{-\alpha}}{\delta y \Gamma(2-\alpha)}\left[v_{i j}^{k-1}\left(u_{i j+1}^{k}-u_{i j}^{k}\right)-\sum_{s=1}^{k-1}\left(\alpha_{s-1}-\alpha_{s}\right) v_{i j}^{k-s-1}\left(u_{i j}^{k-s}-u_{i j-1}^{k-s}\right)\right]+O\left(\tau^{2-\alpha}+\delta y\right) .
\end{array}\right.
$$

Eventually, we obtain the linear iteration scheme and equation for velocity: 
Mathematical Problems in Engineering

13

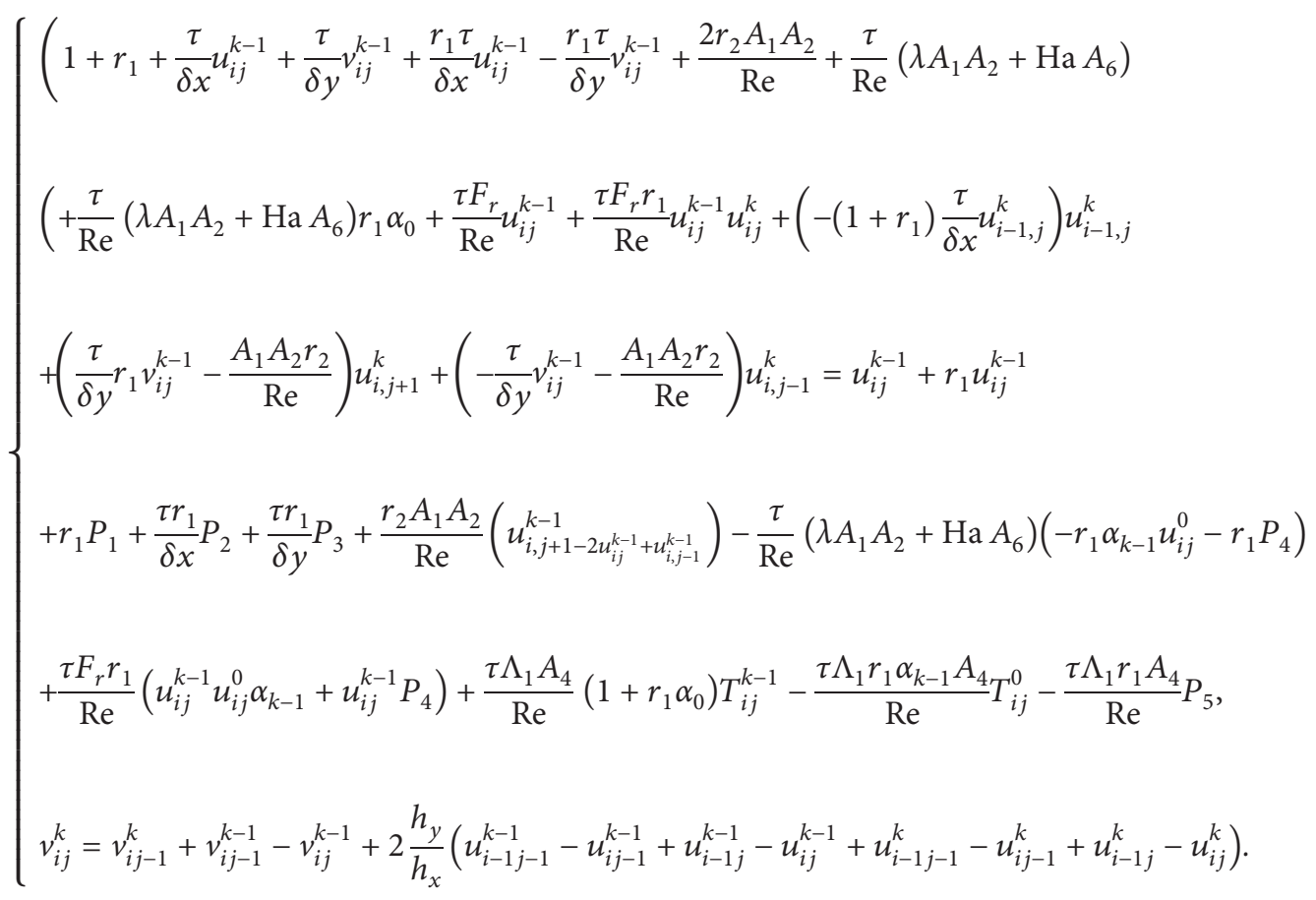

The associated temperature terms are approached by a similar technique of difference. Ultimately, a system of linear iteration equations is acquired:

$$
\left\{\begin{array}{l}
{\left[\left(1+r_{3}\right) \frac{\tau}{\delta x} u_{i j}^{k-1}+\frac{\tau}{\delta y}+\frac{\tau r_{3}}{\delta x} u_{i j}^{k-1}-\frac{\tau r_{3}}{\delta y} v_{i j}^{k-1}+\frac{\tau r_{4}}{(\delta y)^{2}}\right] T_{i j}^{k}+\left[\frac{-\tau}{\delta x} u_{i j}^{k-1}-\frac{\tau r_{3}}{\delta x} u_{i j}^{k-1}\right] T_{i-1, j}^{k}} \\
+\left[\frac{-\tau}{\delta y} v_{i j}^{k-1}-\frac{\tau r_{4}}{2(\delta y)^{2}}\right] T_{i, j+1}^{k}=T_{i j}^{k-1} r_{3} T_{i j}^{k-1}+\frac{\tau r_{4}}{2(\delta y)^{2}}\left[T_{i, j+1}^{k-1}-2 T_{i j}^{k-1}-T_{i j-1}^{k-1}\right]+r_{3} D_{1}+\frac{\tau r_{3}}{\delta x} D_{2}+\frac{\tau r_{3}}{\delta y} D_{3},
\end{array}\right.
$$

where 


$$
\begin{aligned}
& r_{1}=\frac{\lambda_{1}^{\alpha} \tau^{-\alpha}}{\Gamma(2-\alpha)}, \\
& r_{2}=\frac{1}{2(\delta y)^{2}}, \\
& r_{4}=\frac{\lambda_{2}^{\gamma} \tau^{-\gamma}}{\Gamma(2-\gamma)}, \\
& \operatorname{RePr}, \\
& P_{1}=\sum_{s=1}^{k-1}\left(\alpha_{s-1}-\alpha_{s}\right)\left(u_{i j}^{k-s}-u_{i j}^{k-s-1}\right), \\
& P_{2}=\sum_{s=1}^{k-1}\left(\alpha_{s-1}-\alpha_{s}\right) u_{i j}^{k-s-1}\left(u_{i j}^{k-s}-u_{i-1 j}^{k-s}\right), \\
& P_{3}=\sum_{s=1}^{k-1}\left(\alpha_{s-1}-\alpha_{s}\right) v_{i j}^{k-s-1}\left(u_{i j}^{k-s}-u_{i j-1}^{k-s}\right), \\
& P_{4}=\sum_{s=1}^{k-1}\left(\alpha_{s-1}-\alpha_{s}\right)\left(u_{i j}^{k-s}\right), \\
& P_{5}=\sum_{s=1}^{k-1}\left(\alpha_{s-1}-\alpha_{s}\right)\left(T_{i j}^{k-s}\right), \\
& D_{1}=\sum_{s=1}^{k-1}\left(\gamma_{s-1}-\gamma_{s}\right)\left(T_{i j}^{k-s}-T_{i j}^{k-s-1}\right), \\
& D_{3}=\sum_{s=1}^{k-1}\left(\gamma_{s-1}-\gamma_{s}\right) u_{i j}^{k-s-1}\left(T_{j i}^{k-s}-T_{i-1 j}^{k-s}\right), \\
& s=1
\end{aligned}
$$

where $\gamma_{s}=(s+1)^{1-\gamma}-s^{1-\gamma}$, in which $s=0,1,2, \ldots, r$.

Likewise, the thermal condition is computed as follows at $y=0[54]$ :

$$
\begin{aligned}
T_{i, 1}^{k}-T_{i,-1}^{k}+T_{i, 1}^{k-1}-T_{i,-1}^{k-1} & =-4 \delta y(-i \delta x)^{n}, \\
T_{i,-1}^{k}-T_{i,-1}^{k} & =T_{i, 1}^{k}+T_{i, 1}^{k-1}+4 \delta y(-i \delta x)^{n} .
\end{aligned}
$$

For simplification, equation (28) is substituted by iteration equations.

\section{Convergence Analysis}

The numerical convergence of the proposed numerical implicit method will be demonstrated as follows: let $u\left(x_{i}, y_{j}, t_{k}\right)$ and $T\left(x_{i}, y_{j}, t_{k}\right) \quad(i=1,2, \ldots, M x-1 ; j=1,2$, $\ldots, M y-1 ; k=1,2, \ldots, n)$ be the exact solution of the problem (15)-(16) at mesh point $\left(x_{i}, y_{j}, t_{k}\right)$. We considered the problem

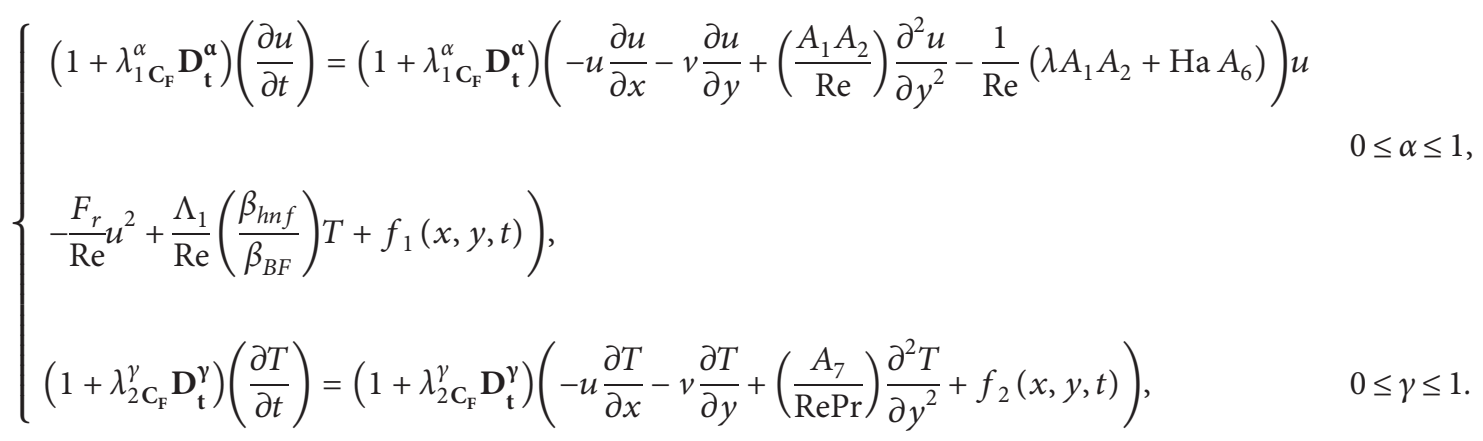


We now considered the boundary conditions for $u$ and $T$ and negligible effect for $v$ since convergence analysis for the above model equations is as follows:

$$
\left\{\begin{array}{l}
u(x, y, 0)=100 y\left(y-L_{y}\right) x\left(x-L_{x}\right), \quad 0 \leq x \leq 1,0 \leq y \leq 1 \\
u(x, 0, t)=0=u\left(x, L_{y}, t\right) \\
u(0, y, t)=0=u\left(L_{x}, y, t\right), \quad 0 \leq t \leq \tau \\
T(x, y, 0)=-t x^{n}\left(x-L_{x}\right), \quad 0 \leq x \leq 1,0 \leq y \leq 1 \\
T(x, 0, t)=0 \\
T\left(x, L_{y}, t\right) \\
T(0, y, t)=0=T\left(L_{x}, y, t\right) \quad 0 \leq t \leq \tau
\end{array}\right.
$$

The right-hand-side terms $f_{1}(x, y, t)$ and $f_{2}(x, y, t)$ are calculated and given in Appendix (A.1). The exact solution is $u(x, y, t)=100\left(1+t^{2}\right) y\left(y-L_{y}\right) x\left(x-L_{x}\right)$ and $T(x, y, t)=$ $t x^{n}(1-y)\left(\left(y-L_{y}\right) / L_{y}\right)\left(x-L_{x}\right)$. Comparison between the exact results and the numerical solutions of $u$ and $T$ are presented in Figure 5 with $\alpha=0.98=\gamma=\lambda_{1}=0.1=\lambda_{2}$, $n=v_{w}=0.1, \mathrm{Ha}=10, \mathrm{Pr}=6.2, F_{r}=\lambda=\Lambda_{1}=0.5, \phi_{\mathrm{Al}_{2} \mathrm{O}_{3}}=$ $0.02=\phi_{\mathrm{ZrO}_{2}}$, and $t=1.0$.The good agreement of results with the experimental data supports the accuracy of our proposed numerical technique.

Moreover, the $L_{2}$ norm is used to calculate the $L_{2}$ error, and analysis of Table 4 shows that the error at $n_{x}$ is minimized by refining mesh size; $n_{y}=20$ corresponds to the optimal error and a $L_{2}$-norm of order $10^{-4}$ and $10^{-5}$. The error is considerably reduced with mesh refinement. The $L_{2}$ norm of the finite difference scheme has a greater order of accuracy for almost all the refinements; however, supreme accuracy is obtained at $n_{x}$ and $n_{y}=200$.

\section{Discussion}

The current section is dedicated to discussing the outcomes acquired when the finite difference method is applied to the discretized momentum and energy equations. The different values of parameters of interest as required in several industrial and biomedical applications are numerically calculated by formulating a MATLAB code. It is shown through graphs followed by a detailed discussion of how the velocity and temperature fields are affected by the fractional derivative, porosity, mass convective parameter, generalized Reynold number, permeable parameter, and power-law exponent. Moreover, a graphical and tabular presentation regarding enhanced thermal conductivity of the proposed hybrid nanofluids over a flat permeable surface is also offered. The impact of nonuniform permeation on the viscoelastic hybrid nanofluid, along with mass transfer over a permeable surface, is also studied in detail. In order to have simplicity, we have fixed the temperature and velocity relaxation times as 0.1. Furthermore, the Reynolds number is assumed to have a fixed value of 0.95 , the Prandtl number is 6.2, and the time is 5.0. The quantities of interest like skin friction coefficient and Nusselt number are evaluated.

The deviation in the velocity of hybrid and single nanofluids against various values of the fractional parameter $\alpha$ is shown in Figure 6(a). A decreasing trend in the velocity of hybrid and single nanofluids is witnessed against an increment in the fractional parameter $\alpha$. At a maximum time $t=5.0$, the behavior of $\alpha$ is analyzed. It is observed that the viscosity of fluids and boundary layer thickness increase against an increment in the value of the fractional parameter, which further results in decreasing the velocity of the fluid. Moreover, a comparative analysis between $\mathrm{Al}_{2} \mathrm{O}_{3}-\mathrm{ZrO}_{2} / \mathrm{BF}$ $(2.0+2.0 \%)$ and the $\mathrm{ZrO}_{2}-\mathrm{BF}(4.0 \%)$ is offered, and it is observed that the velocity of the single nanofluid $\mathrm{ZrO}_{2} / \mathrm{BF}$ faces less viscosity effect than that of the hybrid nanofluid. Furthermore, it is shown that an increment in the fractional parameter $\alpha$ for a small time $(t=0.01)$ significantly contributes to enhancing the velocity of the hybrid nanofluid. The impact of the velocity relaxation parameter on velocity distribution is also attributed through Figure 6(b). This figure also reveals that corresponding to an increase in the relaxation parameter $\lambda_{1}$, the velocity and boundary layer thickness tend to increase and decrease, respectively. The enhanced viscosity of the fluid prevents the occurrence of the delaying characteristic of viscoelastic fluid. Also, in our case, the fluid mixture comprising $\mathrm{Al}_{2} \mathrm{O}_{3}$ and $\mathrm{ZrO}_{2}$ will take maximum time to resume the normal state when the value of $\lambda_{1}$ is increased from 0.0 to 1.2. In addition to this, the graphical presentation gives us a comparative view to witness that hybrid nanofluids assume higher velocity than single nanofluid. Furthermore, the effect of the porosity parameter $\lambda$ on the velocity profile is presented through Figure $6(\mathrm{c})$. It is observed that corresponding to higher values of the porosity parameter $\lambda$, the velocity shows a decreasing trend because the increased value of porosity offers resistance to the flow. On the other hand, corresponding to higher values of the porosity parameter $\lambda$, the boundary layer thickness tends to decrease. The current study also indicates that the viscosity of the fluids increases against an increment in the values of $0 \leq \alpha \leq 1$ and $\lambda_{1}$, which can also restrict the velocity of fluid in the pores of the porous medium. The velocity of the hybrid nanofluid $\mathrm{Al}_{2} \mathrm{O}_{3}-\mathrm{ZrO}_{2} / \mathrm{BF}(2.0+2.0 \%)$ is somewhat slower than that of the single nanofluid $\mathrm{ZrO}_{2}-\mathrm{BF}(4.0 \%)$ as it possess higher density. Along similar lines, corresponding to higher values of the inertia coefficient parameter (Forchheimer parameter) $\left(F_{r}\right)$, the hybrid fluid velocity shows a decreasing behavior (see Figure 6(d)), whereas the thickness of the fluid is not altered by $F_{r}$. Since we have assumed free stream velocity at $y \longrightarrow \infty$, the $F_{r}$ has no impact. Physically, it can be inferred as a state of the porous gap where the pore size is big enough such that the porous medium is stretched with fluid-solid interaction, which further increases the viscous intervention. As better flow resistance is produced against an increase in $F_{r}$, the fluid velocity of both the hybrid nanofluid $\mathrm{Al}_{2} \mathrm{O}_{3}-$ $\mathrm{ZrO}_{2} / \mathrm{BF}(2.0+2.0 \%)$ and single nanofluid $\mathrm{ZrO}_{2}-\mathrm{BF}$ is restricted.

Figure 7 (a) shows the impact of the magnetic parameter $\mathrm{Ha}$ on the velocity profile of both fluids. Here, the Lorentz force is produced, in the presence of a transverse magnetic field, due to the magnetic field's attraction throughout the fluid's motion over a flat permeable surface. The positive values of the magnetic field parameter cause the velocity to 


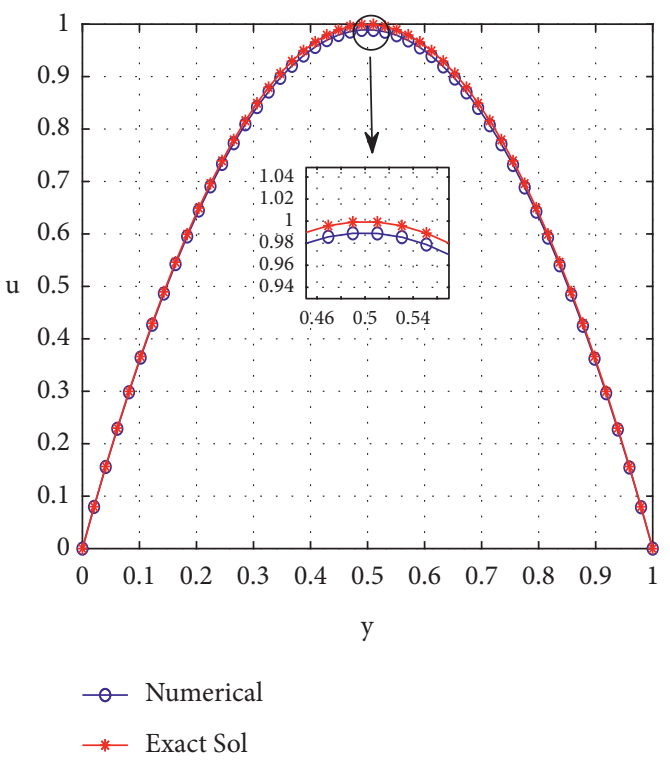

(a)

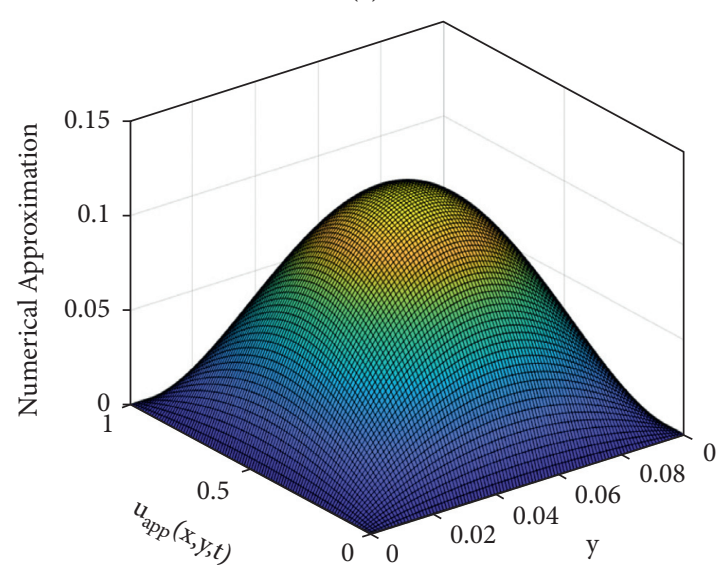

(c)

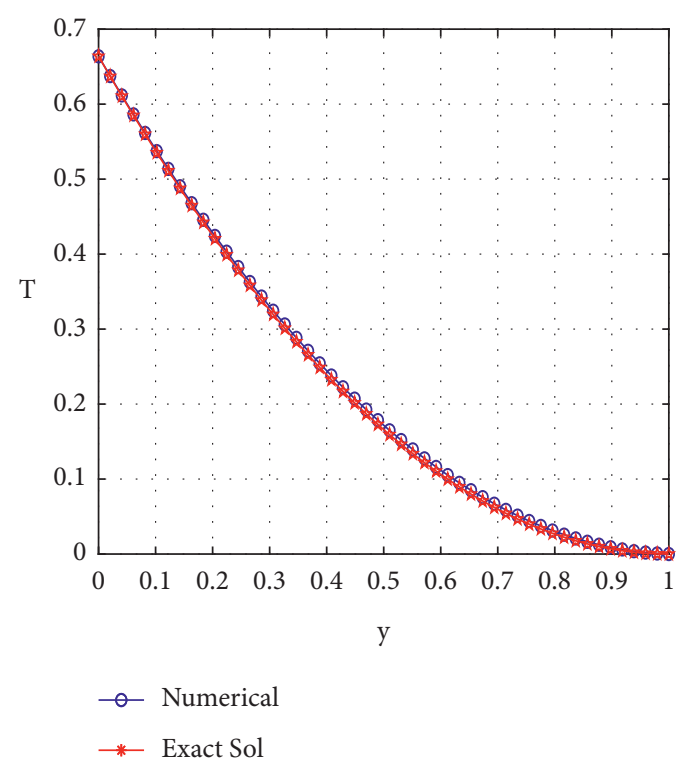

(b)

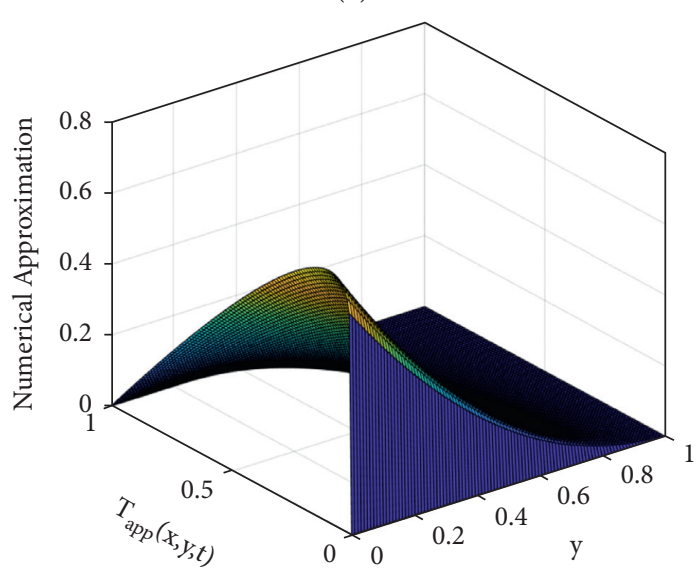

(d)

FIgURE 5: Comparison of exact and numerical solutions for velocity and temperature when $\alpha=0.98=\gamma=, \operatorname{Re}=0.95, \lambda_{1}=$ $0.1=\lambda_{2}, n=v_{w}=0.1, \mathrm{Ha}=10, \mathrm{Pr}=6.2, F_{r}=\lambda=\Lambda_{1}=0.5, \phi_{\mathrm{Al}_{2} \mathrm{O}_{3}}=0.02=\phi_{\mathrm{ZrO}_{2}}$, and $t=1.0$.

TABLE 4: $L_{2}$-norm of the errors in the numerical solutions of the velocity and temperature.

\begin{tabular}{lccc}
\hline Number of mesh points $n_{x}$ along the $x$-axis & Number of $n_{y}$ along the $y$-axis & $\left\|u_{\text {app }}-u_{\text {ex }}\right\|_{L^{2}(\Omega)}$ & $\left\|T_{\text {app }}-T_{\text {ex }}\right\|_{L^{2}(\Omega)}$ \\
\hline 20 & 20 & $11.495 E^{-04}$ & $5.882 E^{-05}$ \\
60 & 60 & $10.729 E^{-04}$ & $5.545 E^{-05}$ \\
100 & 100 & $9.466 E^{-04}$ & $4.949 E^{-05}$ \\
140 & 140 & $8.131 E^{-04}$ & $4.315 E^{-05}$ \\
200 & 200 & $6.088 E^{-04}$ & $3.342 E^{-05}$ \\
\hline
\end{tabular}

decrease because resistive force is increased. Accordingly, the velocity is decreased in the $x$ direction with boundary layer thickness. Upon comparison of velocities of both the nanofluids, it is observed that the resistance forces have a significant impact on the hybrid nanofluid $\mathrm{Al}_{2} \mathrm{O}_{3}-\mathrm{ZrO}_{2} / \mathrm{BF}$ $(2.0+2.0 \%)$ due to its high density than that on the single nanofluid $\mathrm{ZrO}_{2} / \mathrm{BF}$. Several studies suggest different ranges of the parameter like $1.0 \leq \operatorname{Re} \leq 2.0$ and $0.8 \leq \alpha \leq 1.0$. It can be noticed from Figure $7(\mathrm{~b})$ that corresponding to an increment in the generalized Reynolds number ( $R e)$, the velocity shows an increasing trend while a total opposite behavior is witnessed for boundary layer thickness which also agrees with the integer-order model. Hence, we can say that the velocity of the flow can also be characterized by the magnitude of the fractional Reynolds number. Furthermore, the effect of $\Lambda_{1}$ on velocity fields is presented in Figure $7(\mathrm{c})$. In convection problems, the heat transfer is governed by the thermal Grashof number. It can be physically interpreted 


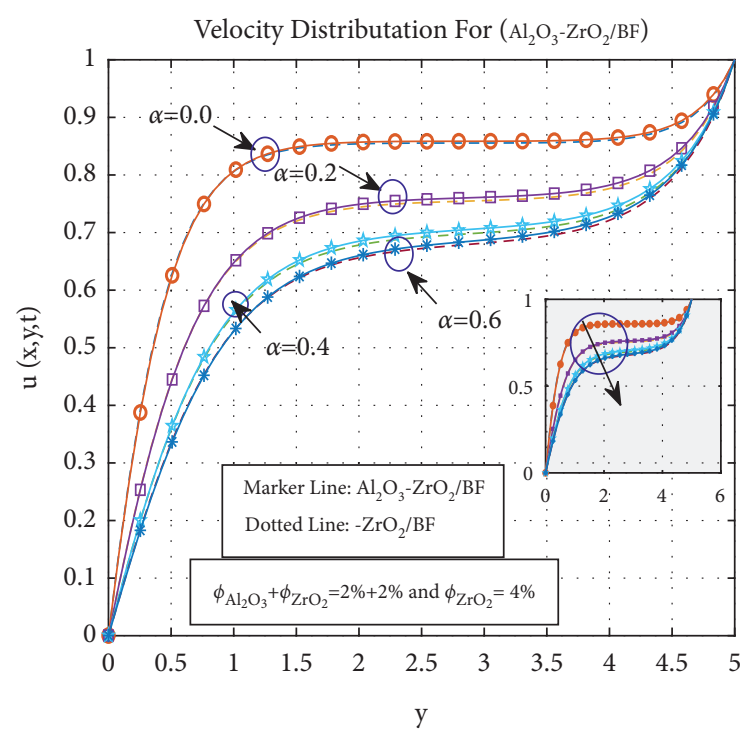

(a)

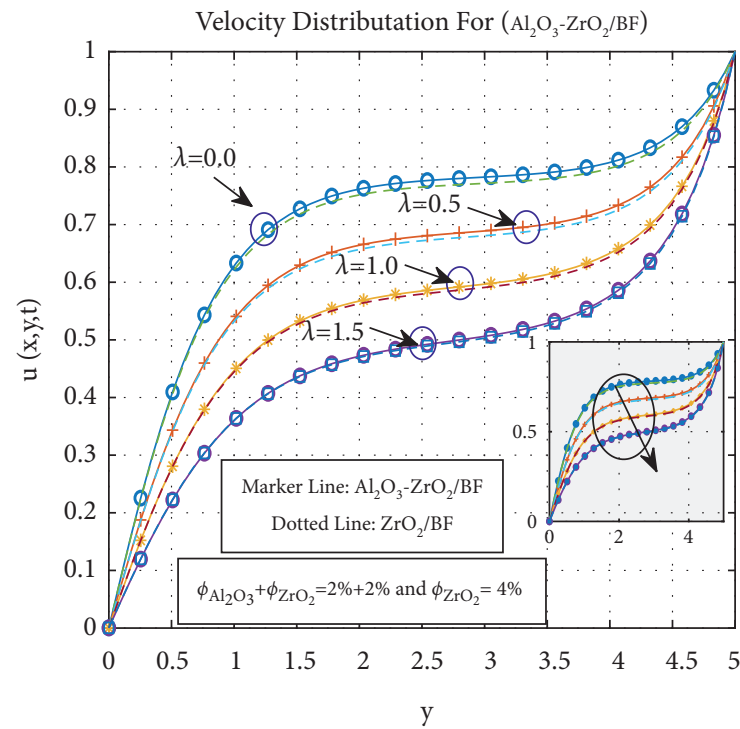

(c)

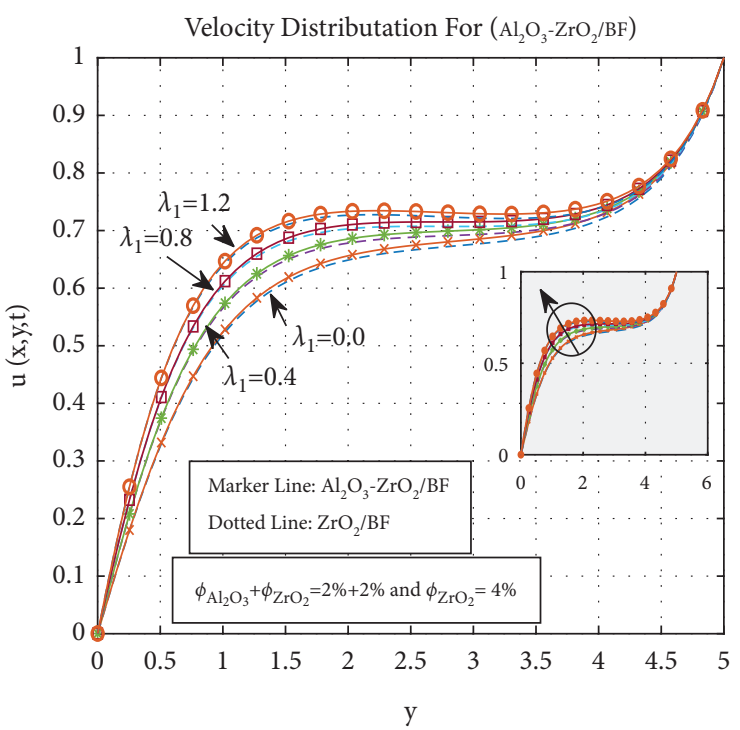

(b)

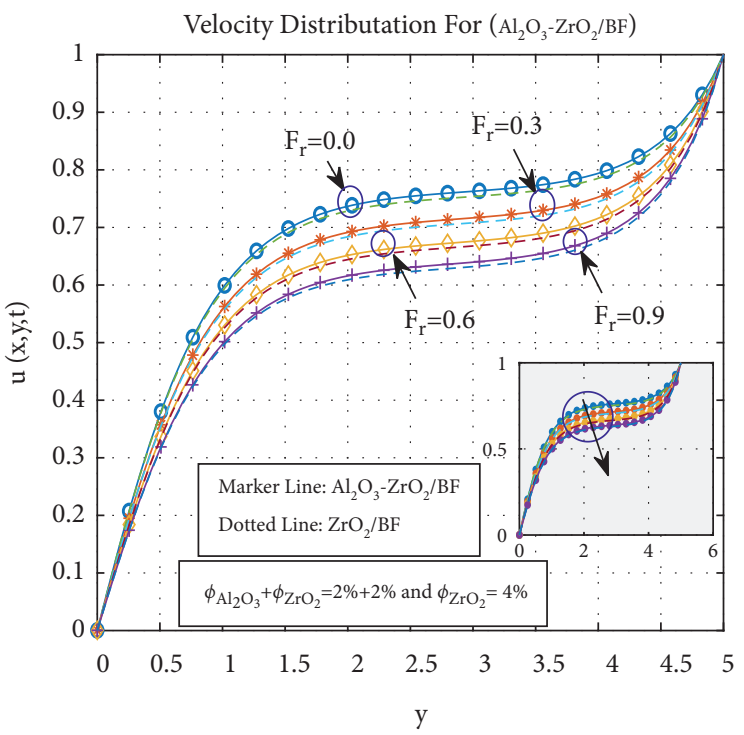

(d)

FIGURE 6: Fractional parameter $\alpha$, velocity relaxation parameter $\lambda_{1}$, porosity parameter $\lambda$, and Forchheimer parameter $F_{r}$ on velocity profile when $\gamma=0.98, \lambda_{2}=0.1, n=v_{w}=0.1, \mathrm{Re}=0.95, \mathrm{Ha}=0.5, \operatorname{Pr}=6.2$, and $t=5.0$.

that positive values of $\Lambda_{1}$ represent heating, while cooling corresponds to negative values. Figure 7(c) clearly shows an increasing trend for velocity profiles in the case of positive values of $\Lambda_{1}$. Because greater values of $\Lambda_{1}$ interpret that magnitude of buoyancy forces is higher, the convection causes an increase in the velocity profiles of both the nanofluids. The enhancement in the velocity of the hybrid nanofluid $\mathrm{Al}_{2} \mathrm{O}_{3}-\mathrm{ZrO}_{2} / \mathrm{BF}(2.0+2.0 \%)$ is greater to some extent when compared with that of the single nanofluid $\mathrm{ZrO}_{2} / \mathrm{BF}$. On the other hand, the velocity may show an opposite trend for negative values of $\Lambda_{1}$ due to the permeable surface's cooling effect. The impact of the permeable parameter on the velocity profile of both the nanofluids is presented in Figure 7(d). It can be seen that change in $v_{w}$ illustrates the improvement in mass transfer via the permeable surface at a low pace where $v_{w}$ is the permeable parameter. In addition to this, it is noted that the optimum permeability of the surface is attained when the length of the permeable plate is fixed. In order to demonstrate the influence of varying heat transports on the boundary layer through the permeable surface, we computed the velocity profile horizontally. We then compared it with that of the conventional layer. Here, we are not comparing vertical velocity as the order of magnitude is not the same as the horizontal velocity. The variation in $u(x, y, t)$ against different permeable parameters $\left(v_{w}\right)$ is reflected through Figure $7(\mathrm{~d})$. It also depicts the change in the horizontal direction with a fixed vertical direction. Figure $7(\mathrm{~d})$ also offers a pictorial view of $u(x, y, t)$-profiles against various permeable parameters $\left(v_{w}\right)$. The function $v_{w}(x)$ increases 


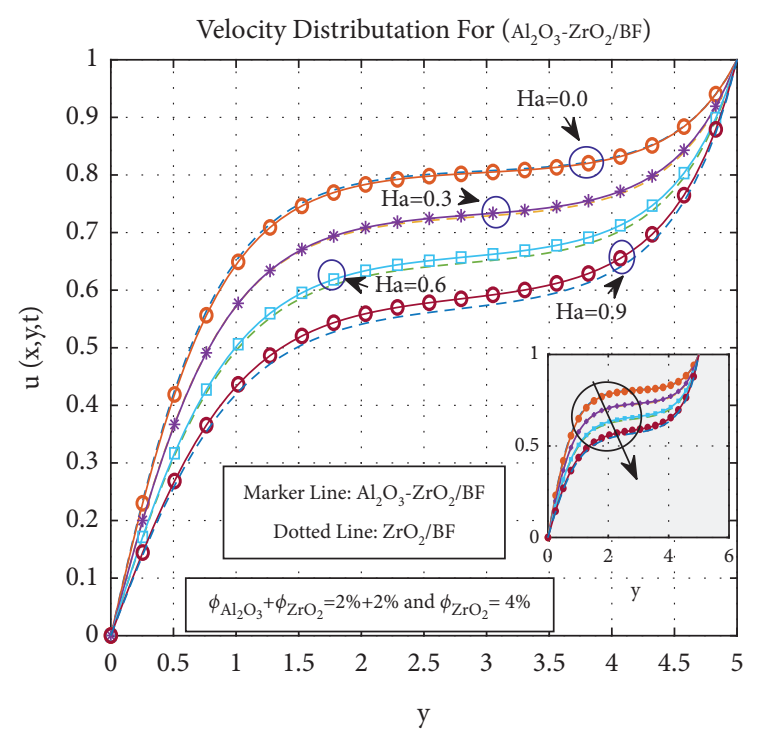

(a)

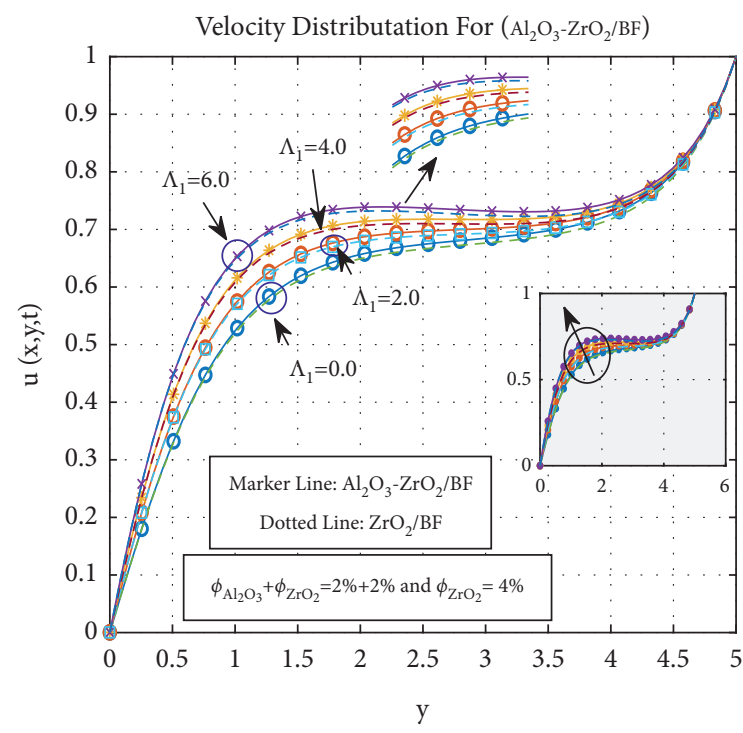

(c)

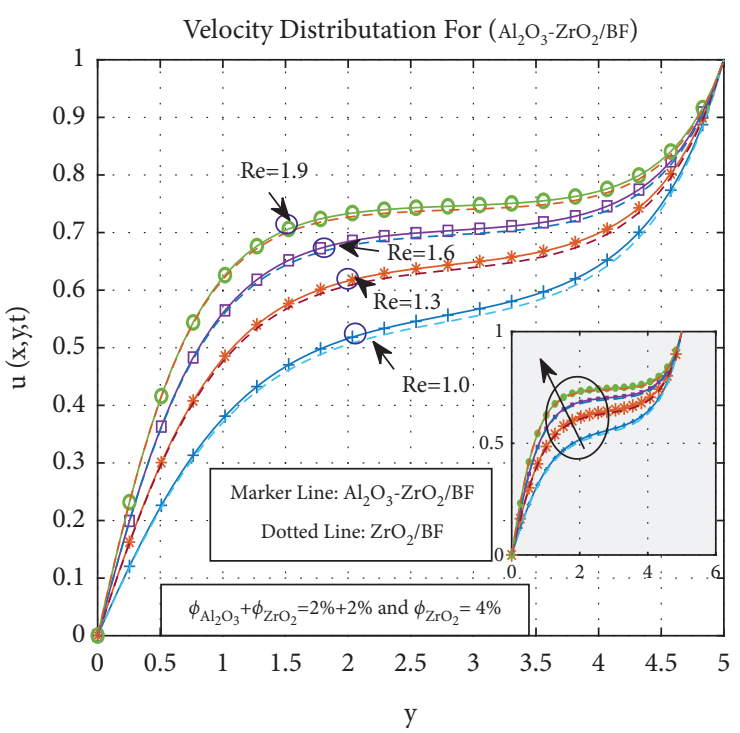

(b)

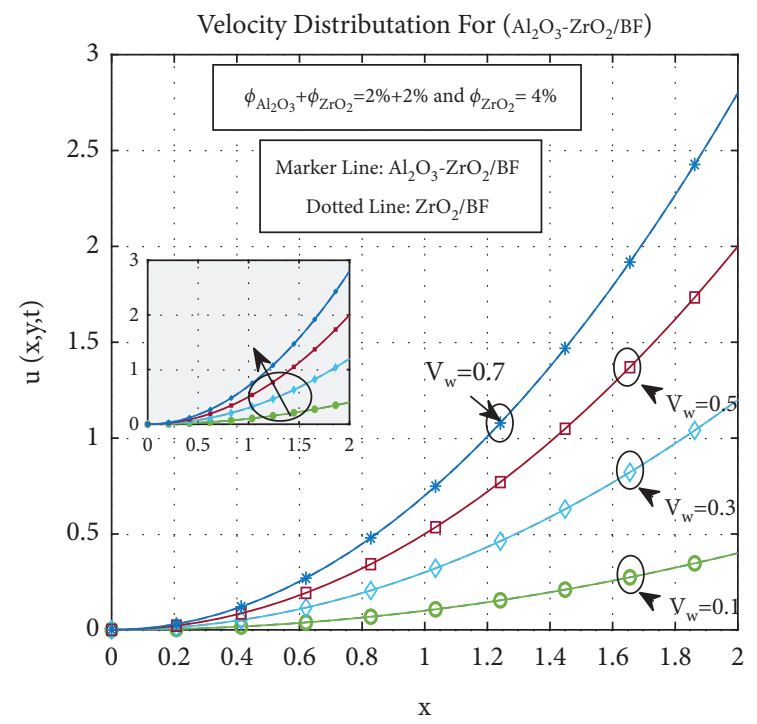

(d)

Figure 7: Hartmann number Ha, generalized Reynolds number Re, Grashof number $\Lambda_{1}$, and permeable parameters $v_{w}$ on the velocity profile when $\alpha=0.98=\gamma, \lambda_{1}=0.1=\lambda_{2}, n=0.1, \operatorname{Pr}=6.2, F_{r}=\lambda=0.5$, and $t=5.0$.

along the surface in the $x$ direction in the case of all the curves. Therefore, the mass transfer is significantly higher via the permeable surface of both the nanofluids and by an increasing $x$. A higher amount of fluid is inoculated into the boundary layer.

How the temperature profile is affected by $\gamma$ is shown through Figure 8(a). A substantial temperature gradient that endorses the convection flow is presented through the smooth profiles. It is also observed that $\gamma$ holds a significant part in the thermal boundary layer as the temperature distribution and the thickness of the thermal boundary layer drastically increase with $\gamma$. The temperature of the hybrid nanofluid $\mathrm{Al}_{2} \mathrm{O}_{3}-\mathrm{ZrO}_{2} / \mathrm{BF}(2.0+2.0 \%)$ is higher to some extent when compared with that of the single nanofluid $\mathrm{ZrO}_{2} / \mathrm{BF}$. The thermal flow is regulated with the help of the order of fractional derivative $(\gamma)$, whereas the velocity flow is controlled by $\alpha$. Moreover, an increment in $\lambda_{2}$ results in a time gap for the transfer of heat to the flow area from the heat source, and resultantly, the temperature profile tends to decrease in that specific region (see Figure 8(b)). In addition, $\lambda_{2}$ and heat transfer rate are directly proportional as the increase in $\lambda_{2}$ results in an increment in the heat transfer rate. Thus, the temperature profile shows a decreasing behavior corresponding to an increase in $\lambda_{2}$. The effect of power-law exponent $n$ on temperature is shown through Figure 8(c). The temperature gradient decreases against an increment in the power-law exponent, and temperature distributions of both nanofluids tend to decline. Figure 8(d) illustrates the effect of Re on the temperature profile. The Reynold number (Re), being one of the most significant parameters in the analysis of heat transfer, also depicts the relative importance of the inertia effect compared to the 


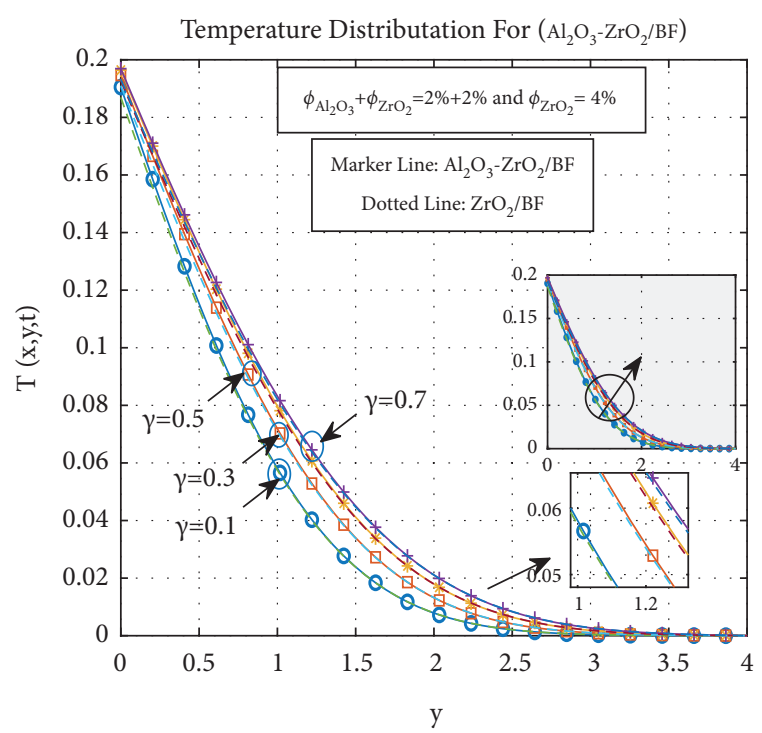

(a)

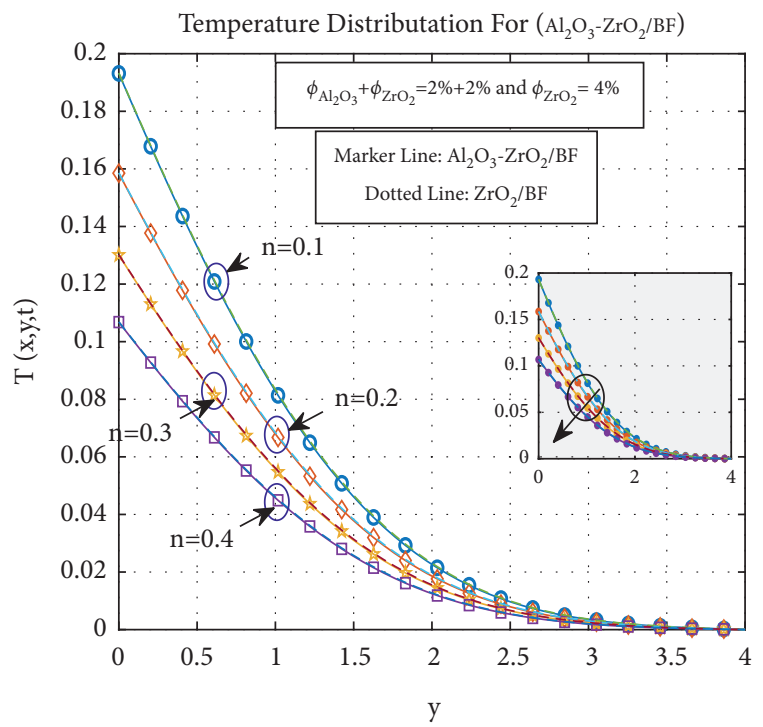

(c)

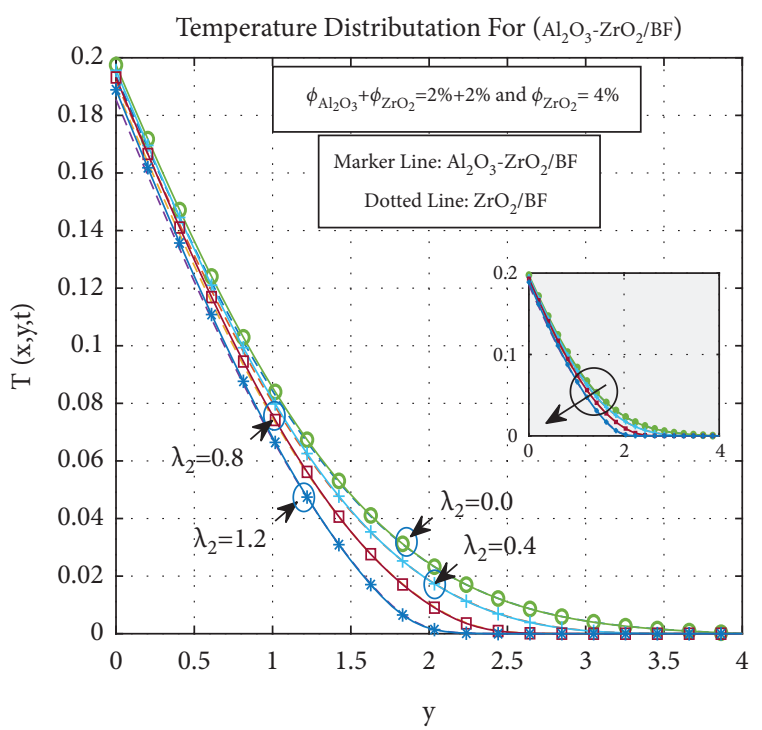

(b)

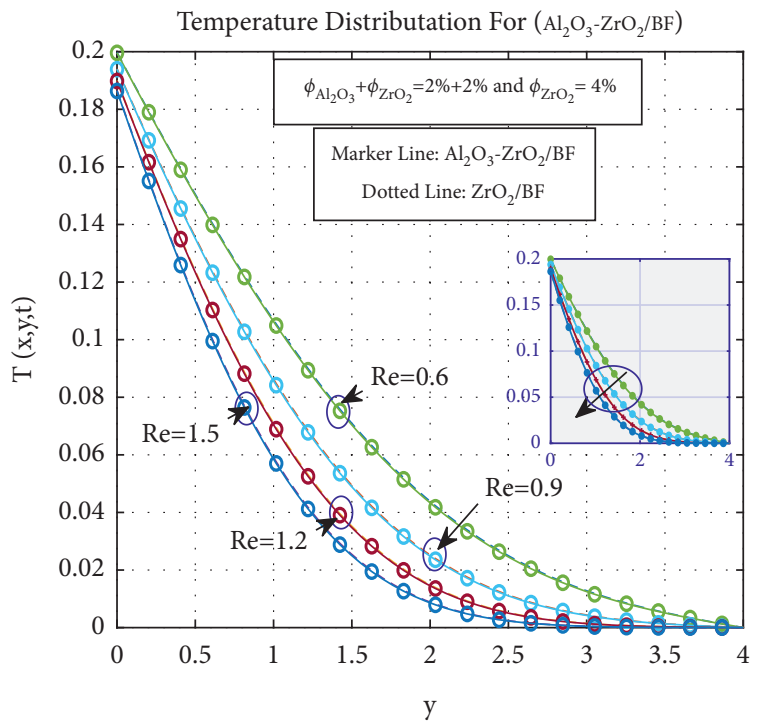

(d)

FIgURE 8: Fractional parameter $\gamma$, temperature relaxation parameter $\lambda_{2}$, power-law index $n$, and generalized Reynolds number Re on the temperature profile when $\alpha=0.98, \lambda_{1}=0.1, \Lambda_{1}=2.0, V_{w}=0.2, \operatorname{Pr}=6.2, F_{r}=\lambda=\mathrm{Ha}=0.5$, and $t=5.0$.

viscous effect. The performance of heat transfer varies at different temperatures, which conveys an evident impact of temperature. In the case of the same nanoparticle volume concentration, the temperature profiles of both the nanofluids tend to decrease when the generalized Reynolds number $(\mathrm{Re})$ is increased. Consequently, the extent to which the heat transfer can be enhanced is extended.

The results obtained in the current study suggest that our proposed numerical approach is efficient irrespective of the permeation function. Hence, a comparison of the three different permeation functions with the same permeable parameter $\left(v_{w}\right)$ is offered for both the hybrid and single nanofluids in Figure 9(a). Furthermore, we have assumed the three parameters $\left(v_{w}(x)\right)$ as follows: The three different scenarios are as follows. Case I: $v_{w}(x)=v_{w} \cdot x$, a basic function that deals with permeability at the surface; Case II: $v_{w}(x)=v_{w} \cdot x^{2}$, a quadratic function that presents a precise nonlinear permeability at the surface; Case III: $v_{w}(x)=v_{w} \cdot \sin (\Pi \cdot x / 2)$, a well-known permeability function that is considered in a number of numerical studies performed earlier. Figure 9(a) shows the impact of the nonuniform permeable sheet on the velocity boundary layer. In real-world problems, this phenomenon occurs due to an anisotropic membrane. Moreover, the velocity distribution in the boundary layer can be altered by changing the permeability on the surface. The said technique makes the current approach different from the other classic methods where inlet velocity, the Reynolds number (Re), or the physical properties of the fluids are altered to change the velocity. In the current study, we have assumed three 


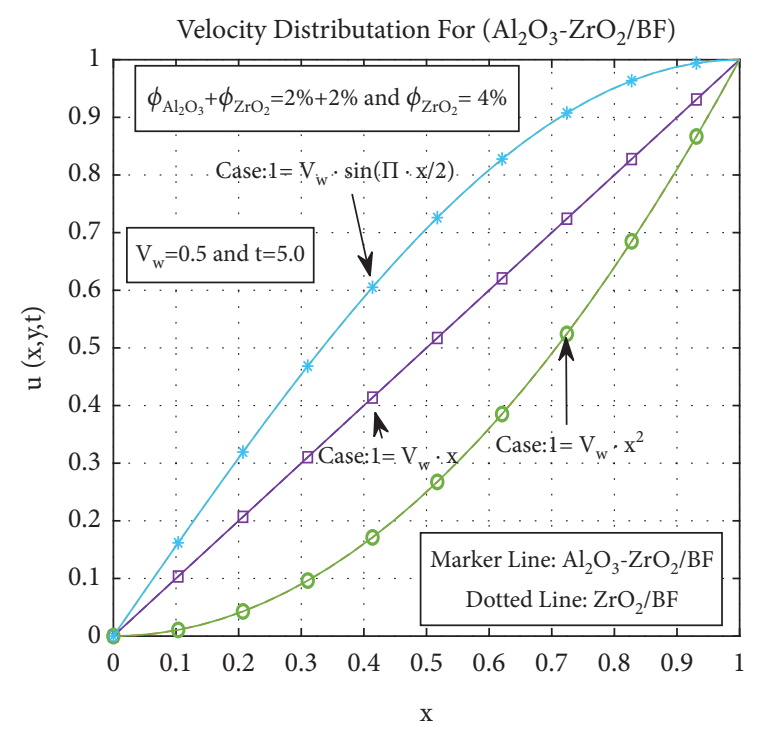

(a)

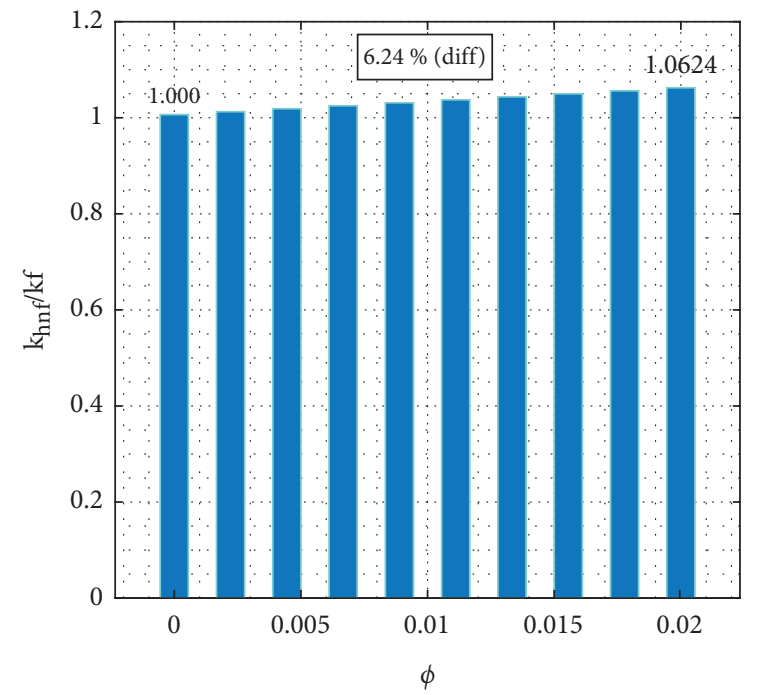

(b)

FIGURE 9: Different permeable situations $v_{w}$ on the velocity profile and thermal conductivity measured for the hybrid water-based suspensions of $\mathrm{Al}_{2} \mathrm{O}_{3}-\mathrm{ZrO}_{2} / \mathrm{BF}$ nanoparticles when $\alpha=0.98=\gamma, \lambda_{1}=0.1=\lambda_{2}, \mathrm{Ha}=0.5, n=0.1, \operatorname{Pr}=6.2, F_{r}=\lambda=0.5$, and $t=5.0$.

TABle 5: Comparison between the predicted thermal conductivity from different models (correlations) and the proposed correlation.

\begin{tabular}{|c|c|c|c|c|c|}
\hline Nanoparticle volume fraction & 0 & 0.005 & 0.01 & 0.015 & 0.02 \\
\hline Present study & 1 & 1.0210 & 1.0346 & 1.0480 & 1.0624 \\
\hline Yu and Choi [64] (\% diff) & 1 & $1.0170(0.4 \%)$ & $1.0340(0.06 \%)$ & $1.0530(0.4 \%)$ & $1.0710(0.8 \%)$ \\
\hline Hamilton and Crosser [65] (\% diff) & 1 & $1.0140(0.7 \%)$ & $1.0290(0.55 \%)$ & $1.0440(0.3 \%)$ & $1.0590(0.3 \%)$ \\
\hline Prasher et al. [66] (\% diff), equations (1) and (2) & 1 & $1.0140(0.7 \%)$ & $1.0290(0.55 \%)$ & $1.0440(0.3 \%)$ & $1.0590(0.3 \%)$ \\
\hline Jan et al. [63] (\% diff) & 1 & $1.0470(2.5 \%)$ & $1.0940(5.70 \%)$ & $1.1240(7.2 \%)$ & $1.1580(8.9 \%)$ \\
\hline
\end{tabular}

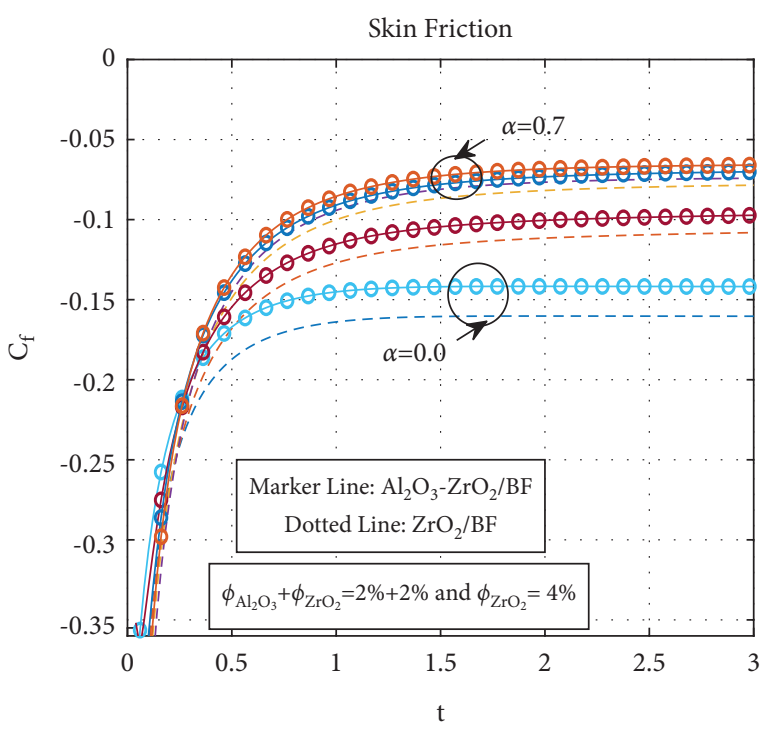

(a)

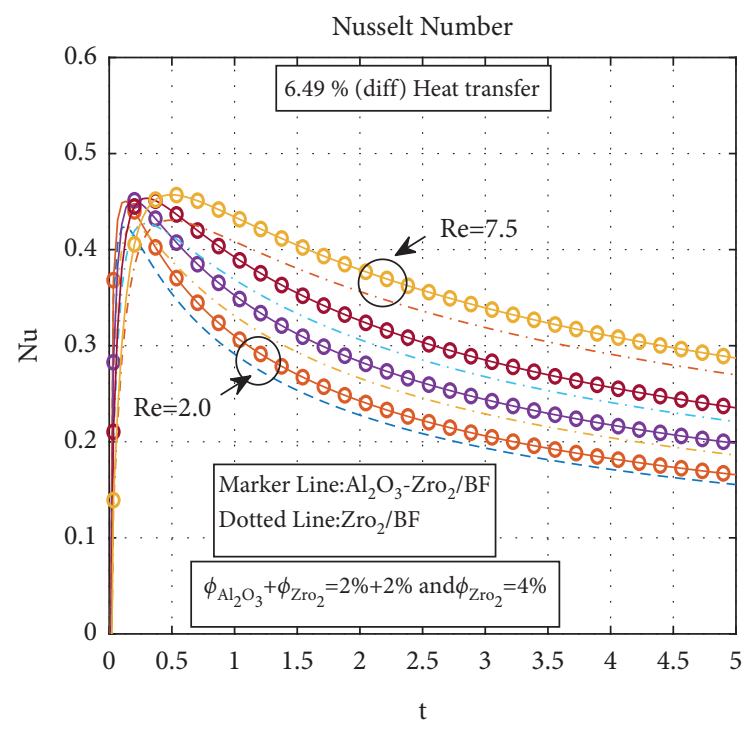

(b)

FIgURE 10: Average skin friction coefficient and Nusselt number distributions for different $\alpha$ and $\operatorname{Re}$ for $\mathrm{Al}_{2} \mathrm{O}_{3}-\mathrm{ZrO}_{2} / \mathrm{BF}$ nanoparticles when $\gamma=0.5, \lambda_{1}=0.01=\lambda_{2}, \mathrm{Ha}=5.5, n=0.01, \operatorname{Pr}=6.2, F_{r}=\lambda=0.5$, and $t=3.0$ and 5.0. 
different permeation functions following the identical pattern where the permeability at the right side of the surface differs from that at the left side. We have assumed the same pattern for comparison purposes. Figure 9(b) suggests that against an increment in the volume concentration of the nanoparticles, the thermal conductivity of the hybrid nanofluid shows an increasing and linear trend. It is also noticed that the hybridization of the nanofluid considerably increases the thermal conductivity.

The variance in viscosity between $\mathrm{Al}_{2} \mathrm{O}_{3}-\mathrm{ZrO}_{2} / \mathrm{BF}$ and $\mathrm{ZrO}_{2} / \mathrm{BF}$ is widened with concentration of nanoparticles. Moreover, Table 5 illustrates that the thermal conductivity is being underrated by all these models when compared to the acquired correlation and the variation becomes more substantial with enhanced nanoparticle volume concentration. The table, as mentioned earlier, also offers a numerical comparison between calculated values of thermal conductivity ratios in [63] and the values projected by many other models. It can be noticed that the increment in gradient responds to an increase in nanoparticle concentration. Figure 10 presents the local skin friction coefficient and Nusselt number distributions against different values of $\alpha$ and Re. It can be witnessed that both the surface tension and local Nusselt number tend to increase against an increment in fractional derivative and generalized Reynold number. Furthermore, an important observation is witnessed that the surface tension shows an increasing behavior. At the same time, the velocity exhibits a decreasing trend for the value of $\alpha$ due to the high viscosity fluid effect. Similarly, the heat transfer rate shows a considerable increase corresponding to an increase in the generalized Reynold number Re. Objectively, it can be said that the heat transfer is increased to $74 \%$ as compared to a single nanofluid where the heat transfer rate escalates but is slower than that of hybrid nanofluid.

\section{Conclusion}

Heat efficiency and heat storage always remain as appealing subjects for researchers when it comes to renewable energy. Various fluid classes have been offered, and several types of phase-change materials have been utilized for energy storage. Nanofluids, liquids having nonmetallic particles, for instance, water, have been gaining significant attention in the recent past. In the current article, the application of fractional calculus is elaborated and fractional partial differential equations governing the mixed convection flow of hybrid nanofluids over a semi-infinite permeable surface subject to the applied magnetic field and variable heat flux are offered. The hybrid nanofluid is prepared with dispersion of nanoparticles $\left(\mathrm{Al}_{2} \mathrm{O}_{3}\right.$ and $\left.\mathrm{ZrO}_{2}\right)$ in a viscoelastic surfactant solution, aqueous solution of cetyltrimethylammonium chloride (CTAC) and sodium salicylate (NaSal). Furthermore, we have considered a flat surface filled with a porous medium, and the notion of Caputo fractional derivatives is used. Commercial aluminum and zirconium particles, having diameters of $<50 \mathrm{~nm}$ and $<20 \mathrm{~nm}$, respectively, were distributed in water. The solution of the governing equations is determined with the help of the finite difference method and the $L_{1}$ algorithm. Furthermore, a detailed analysis of the effects of parameters of interest on velocity and temperature fields is offered. The following explicit and important inferences are obtained from the current numerical study:

(i) Variation in the magnetic parameter together with the inertia coefficient $F_{r}$ and simultaneously changing generalized Reynold number Re have caused variation in the approximate solutions for both velocity and temperature equations, and these variations follow the same pattern as obtained from different numerical schemes, which endorses the accuracy of our proposed scheme. Thus, our proposed numerical scheme can be referred to as an alternate and efficient solver.

(ii) Comparative analysis of the offered scheme suggests the accuracy of $10^{-04}$ for the solution $u(x, y, t)$ when the mesh size is increased along $x$ and $y$ directions. In contrast, the accuracy for the solutions of $T(x, y, t)$ is somewhat near $10^{-05}$.

(iii) The temperature profiles considerably increase against an increase in fractional derivative parameters $(\gamma)$, and accordingly, the viscoelastic effects of the fluids are enhanced. A remarkable rise in temperature is observed for $\mathrm{Al}_{2} \mathrm{O}_{3}-\mathrm{ZrO}_{2} / \mathrm{BF}$ as compared with $\mathrm{ZrO}_{2} / \mathrm{BF}$.

(iv) Furthermore, when the generalized Reynolds number $(\mathrm{Re})$ is increased, the temperature profile of both nanofluids decreases and hence produces the optimum level of heat transfer over the permeable surface.

(v) In investigating the effect of nanoparticles, hybrid nanoparticles have had a more efficient role in heat transfer enhancement than a single nanofluid over a nonuniform permeable surface.

(vi) During the analysis of the impact of nanoparticles, it is revealed that hybrid nanoparticles play a more dominant role in enhancing heat transfer than single nanoparticles over a nonuniform permeable surface. It is noted that the thermal conductivity of nanofluid $\mathrm{Al}_{2} \mathrm{O}_{3}-\mathrm{ZrO}_{2} / \mathrm{BF}$ is increased by $6.24 \%$ (diff) when the volume concentration is increased.

(vii) A visible increment in heat transfer rate is observed with an increase in Reynold number Re for hybrid and single nanofluids. The average skin friction coefficient follows the same trend when the value of $\alpha$ is increased.

Finally, it is established from the data analysis that hybrid nanofluids have significant implications in the applications of thermodynamics along with considerable scale control over the behavior of the synthetic fluid. Considering a precise application and particular engineering nanofluids for that application, the hybrid nanofluids can entirely change the field of heat transfer. Thus, we can say that in order to have a more efficient thermal system, it is suggested 
that hybrid nanofluids should be used instead of a single type of nanoparticle. Thus, the challenge of manufacturing Appendix economic and stable products can be more efficiently addressed, leading to novel research avenues.

$$
\begin{aligned}
f_{1}(x, y, t) & \\
& +y^{2}\left(y-L_{y}\right)^{2} x\left(x-L_{x}\right)\left(2 x-L_{x}\right)\left(\left(1+t^{2}\right)^{2}+\frac{24 \lambda_{1}^{\alpha} t^{4-\alpha}}{\Gamma(5-\alpha)}+\frac{4 \lambda_{1}^{\alpha} t^{2-\alpha}}{\Gamma(3-\alpha)}\right)-\left(\frac{2 A_{1} A_{2}}{\operatorname{Re}}\right)\left(1+t^{2}\right)^{2} x\left(x-L_{x}\right) \\
& +\left(\lambda A_{1} A_{2}+\operatorname{Ha} A_{6}\right) y\left(y-L_{y}\right) x\left(x-L_{x}\right)\left(\left(1+t^{2}\right)+\frac{2 \lambda_{1}^{\alpha} t^{2-\alpha}}{\Gamma(3-\alpha)}\right)+\frac{F_{r}}{\operatorname{Re}} y^{2}\left(y-L_{y}\right)^{2} x^{2}\left(x-L_{x}\right)^{2} \\
& \left.\cdot\left(\left(1+t^{2}\right)^{2}+\frac{24 \lambda_{1}^{\alpha} t^{4-\alpha}}{\Gamma(5-\alpha)}+\frac{4 \lambda_{1}^{\alpha} t^{2-\alpha}}{\Gamma(3-\alpha)}\right)\right) \\
f_{2}(x, y, t)= & x^{n}(1-y) \frac{\left(y-L_{y}\right)}{L_{y}}\left(x-L_{x}\right)+\left(t+t^{3}\right) x\left(L_{x}-x\right) y\left(L_{y}-y\right)\left((n+1) x^{n}-L_{x} n x^{n-1}\right)(1-y) \frac{\left(y-L_{y}\right)}{L_{y}} \\
& +\frac{\lambda_{2}^{\gamma} t^{1-\gamma}}{\Gamma(2-\gamma)} x^{n}\left(x-L_{x}\right)\left(y-L_{y}\right)(1-y)+\lambda_{2}^{\gamma} x\left(L_{x}-x\right) y\left(L_{y}-y\right)\left((n+1) x^{n}-L_{x} n x^{n-1}\right) \\
& \cdot(1-y) \frac{\left(y-L_{y}\right)}{L_{y}}\left(\frac{t^{1-\gamma}}{\Gamma(2-\gamma)}+\frac{6 t^{3-\gamma}}{\Gamma(4-\gamma)}\right)+\left(\frac{A_{7}}{\operatorname{RePr}}\right) \frac{2 t x^{n}\left(x-L_{x}\right)}{L_{y}} .
\end{aligned}
$$

\section{Data Availability}

The data used to support the findings of this study are included within the article.

\section{Conflicts of Interest}

The authors declare no conflicts of interest.

\section{Authors' Contributions}

Both authors have contributed to writing and proofreading of the manuscript.

\section{References}

[1] H. E. Mohammad, B. Mehdi, H. Hamid, and V. Majid, "A comprehensive review on convective heat transfer of nanofluids in porous media: energy-related and thermohydraulic characteristics," Applied Thermal Engineering, vol. 178, Article ID 115487, 2020.

[2] B. Shahram, S. Hossein, S. M. A. Seyed, and N. Mohammad, "An experimental investigation on forced convection heat transfer of single-phase flow in a channel with different arrangements of porous media," International Journal of Thermal Sciences, vol. 134, pp. 370-379, 2018.

[3] M. Sheikholeslami, "Application of Darcy law for nanofluid flow in a porous cavity under the impact of Lorentz forces," Journal of Molecular Liquids, vol. 266, pp. 495-503, 2018.

[4] M. Sheikholeslami, "New computational approach for exergy and entropy analysis of nanofluid under the impact of Lorentz force through a porous media," Computer Methods in Applied Mechanics and Engineering, vol. 344, pp. 319-333, 2019.

[5] L. H. Ya, L. Qing, L. Qing, and Q. T. Wen, "Lattice Boltzmann methods for single-phase and solid-liquid phase-change heat transfer in porous media: a review," International Journal of Heat and Mass Transfer, vol. 129, pp. 160-197, 2019.

[6] K. Alibakhsh, D. Reza, M. Omid et al., "Nanofluid flow and heat transfer in porous media: a review of the latest developments," International Journal of Heat and Mass Transfer, vol. 107, pp. 778-791, 2017.

[7] B. T. Farzad, K. Arash, and I. V. Seyed, "Flow and heat transfer analysis of $\mathrm{TiO}_{2}$ /water nanofluid in a ribbed flat-plate solar collector," Renewable Energy, vol. 122, pp. 406-418, 2018.

[8] T. Hayat, M. Khan, T. Muhammad, and A. Alsaedi, "A useful model for squeezing flow of nanofluid," Journal of Molecular Liquids, vol. 237, pp. 447-454, 2017.

[9] B. Mehdi and H. Saeed, "Efficacy of a novel liquid block working with a nanofluid containing graphene nanoplatelets decorated with silver nanoparticles compared with conventional cpu coolers," Applied Thermal Engineering, vol. 127, pp. 1233-1245, 2017.

[10] A. J. Chamkha, M. Molana, A. Rahnama, and F. Ghadami, "On the nanofluids applications in microchannels: a comprehensive review," Powder Technology, vol. 332, pp. 287-322, 2018.

[11] S. U. S. Choi, "Enhancing thermal conductivity of fluids with nanoparticles," ASME FED, vol. 66, pp. 99-105, 1995

[12] S. U. S. Choi, Z. G. Zhang, W. Yu, F. E. Lockwood, and E. A. Grulke, "Anomalous thermal conductivity enhancement in nanotube suspensions," Applied Physics Letters, vol. 79, no. 14 , pp. 2252-2254, 2001. 
[13] S. Lei, H. Yurong, H. Yanwei, and W. Xinzhi, "Thermophysical properties of $\mathrm{Fe}_{3} \mathrm{O}_{4} @ \mathrm{CNT}$ nanofluid and controllable heat transfer performance under magnetic field," Energy Conversion and Management, vol. 177, pp. 249-257, 2018.

[14] M. Sheikholeslami, M. Jafaryar, E. Abohamzeh, A. Shafee, and H. Babazadeh, "Energy and entropy evaluation and two-phase simulation of nanoparticles within a solar unit with impose of new turbulator," Sustainable Energy Technologies and Assessments, vol. 39, Article ID 100727, 2020.

[15] M. Sheikholeslami, S. A. M. Mehryan, A. Shafee, and M. A. Sheremet, "Variable magnetic forces impact on magnetizable hybrid nanofluid heat transfer through a circular cavity," Journal of Molecular Liquids, vol. 277, pp. 388-396, 2019.

[16] H. Xie, J. Wang, T. Xi, and Y. Liu, "Thermal conductivity of suspensions containing nanosized SiC particles," International Journal of Thermophysics, vol. 23, no. 2, pp. 571-580, 2002.

[17] G. Zyla and J. Fal, "Viscosity, thermal and electrical conductivity of silicon dioxide-ethylene glycol transparent nanofluids: an experimental studies," Thermochimica Acta, vol. 650, pp. 106-113, 2017.

[18] A. Naddaf and S. Zeinali Heris, "Experimental study on thermal conductivity and electrical conductivity of diesel oilbased nanofluids of graphene nanoplatelets and carbon nanotubes," International Communications in Heat and Mass Transfer, vol. 95, pp. 116-122, 2018.

[19] L. Yang and X. Xu, "A renovated Hamilton-crosser model for the effective thermal conductivity of CNTs nanofluids," International Communications in Heat and Mass Transfer, vol. 81, pp. 42-50, 2017.

[20] T. Hayat, M. Khan, M. Taseer, and A. Alsaedi, "On model for three-dimensional flow of nanofluid with heat and mass flux boundary conditions," Journal of Molecular Liquids, vol. 10, no. 3, Article ID 031003, 2018.

[21] N. Kumar, S. S. Sonawane, and S. H. Sonawane, "Experimental study of thermal conductivity, heat transfer and friction factor of $\mathrm{AL}_{2} \mathrm{O}_{3}$ based nanofluid," International Communications in Heat and Mass Transfer, vol. 90, pp. 1-10, 2018.

[22] H. E. Mohammad, F. Masoumeh, and A. Masoud, "Experimental and theoretical investigation of thermal conductivity of ethylene glycol containing functionalized single walled carbon nanotubes," Physica E: Low-dimensional Systems and Nanostructures, vol. 95, pp. 71-77, 2018.

[23] A. Tanveer, T. Salahuddin, M. Khan, A. S. Alshomrani, and M. Y. Malik, "The assessment of nanofluid in a von karman flow with temperature relied viscosity," Results in Physics, vol. 9, pp. 916-922, 2018.

[24] I. Haider, U. Nazir, M. Nawaz, S. O. Alharbi, and I. Khan, "Numerical thermal study on performance of hybrid nanowilliamson fluid with memory effects using novel heat flux model," Case Study Thermal Engineering, vol. 26, Article ID 101070, 2021.

[25] M. Khan and A. Rasheed, "Slip velocity and temperature jump effects on molybdenum disulfide MoS2 and silicon oxide $\mathrm{SiO} 2$ hybrid nanofluid near irregular 3D surface," Alexandria Engineering Journal, vol. 60, no. 1, pp. 1689-1701, 2021.

[26] A. Shafiq, S. A. Lone, T. N. Sindhu, Q. M. Al-Mdallal, and G. Rasool, "Statistical modeling for bioconvective tangent hyperbolic nanofluid towards stretching surface with zero mass flux condition," Scientific Reports, vol. 11, no. 1, p. 13869, 2021.
[27] A. Ahangarpour and M. Farbod, "The noble effect of aging on the thermal conductivity of modified CNTs-ethylene glycol nanofluids," Physics and Chemistry of Liquids, vol. 56, no. 9-15, 2018.

[28] B. Bahaya, D. Johnson, and C. Yavuzturk, "On the effect of graphene nanoplatelets on water-graphene nanofluids thermal conductivity, viscosity, and heat transfer under laminar external flow conditions," Journal of Heat Transfer, vol. 140, Article ID 064501, 2018.

[29] M. Hemmat Esfe, M. Firouzi, and M. Afrand, "Experimental and theoretical investigation of thermal conductivity of ethylene glycol containing functionalized single walled carbon nanotubes," Physica E: Low-Dimensional Systems and Nanostructures, vol. 95, pp. 71-77, 2018.

[30] S. A. M. Mehryan, M. A. Sheremet, M. Soltani, M. Izadi, and I. Mohsen, "Natural convection of magnetic hybrid nanofluid inside a double-porous medium using two-equation energy model," Journal of Molecular Liquids, vol. 277, pp. 959-970, 2019.

[31] M. Ghalambaz, A. J. Chamkha, and D. Wen, "Natural convective flow and heat transfer of nano-encapsulated phase change materials (NEPCMs) in a cavity," International Journal of Heat and Mass Transfer, vol. 138, pp. 738-749, 2019.

[32] S. Mehryan, E. Izadpanahi, M. Ghalambaz, and A. J. Chamkha, "Mixed convection flow caused by an oscillating cylinder in a square cavity filled with $\mathrm{Cu}-\mathrm{Al}_{2} \mathrm{O}_{3}$ /water hybrid nanofluid," Journal of Thermal Analysis and Calorimetry, vol. 137, 2019.

[33] T. Tayebi and A. J. Chamkha, "Magnetohydrodynamic natural convection heat transfer of hybrid nanofluid in a square enclosure in the presence of a wavy circular conductive cylinder," Journal of Thermal Science and Engineering Applications, vol. 12, 2019.

[34] S. Aman, Q. Al-Mdallal, and I. Khan, "Heat transfer and second order slip effect on MHD flow of fractional Maxwell fluid in a porous medium," Journal of King Saud University Science, vol. 32, no. 1, pp. 450-458, 2020.

[35] S. Aman, I. Khan, Z. Ismail, M. Z. Salleh, A. S. Alshomrani, and M. S. Alghamdi, "Magnetic field effect on Poiseuille flow and heat transfer of carbon nanotubes along a vertical channel filled with Casson fluid," AIP Advances, vol. 7, no. 1, Article ID 015036, 2017.

[36] M. Hemmat Esfe, S. Esfandeh, and M. Rejvani, "Modeling of thermal conductivity of MWCNT-SiO2 (30:70\%)/EG hybrid nanofluid, sensitivity analyzing and cost performance for industrial applications," Journal of Thermal Analysis and Calorimetry, vol. 131, no. 2, pp. 1437-1447, 2018.

[37] L. Haoran, W. Li, H. Yurong, H. Yanwei, Z. Jiaqi, and J. Baocheng, "Experimental investigation of thermal conductivity and viscosity of ethylene glycol based $\mathrm{ZnO}$ nanofluids," Applied Thermal Engineering, vol. 88, pp. 363-368, 2015.

[38] M. Mehdi and A. Masoud, "Thermal conductivity enhancement of $\mathrm{COOH}$-functionalized MWCNTs/ethylene glycol-water nanofluid for application in heating and cooling systems," Applied Thermal Engineering, vol. 105, pp. 716-723, 2016.

[39] E. A. Sameh, "Caputo fractional convective flow in an inclined wavy vented cavity filled with a porous medium using $\mathrm{AL}_{2} \mathrm{O}_{3}$ $\mathrm{Cu}$ hybrid nanofluids," International Communications in Heat and Mass Transfer, vol. 116, Article ID 104690, 2020.

[40] E. Barkai, R. Metzler, and J. Klafter, "From continuous time random walks to the fractional Fokker-Planck equation," Physical Review, vol. 61, no. 1, pp. 132-138, 2000. 
[41] V. V. Kulish and J. L. Lage, "Application of fractional calculus to fluid mechanics," Journal of Fluids Engineering, vol. 124, no. 3, pp. 803-806, 2002.

[42] R. L. Magin, O. Abdullah, D. Baleanu, and X. J. Zhou, "Anomalous diffusion expressed through fractional order differential operators in the Bloch-Torrey equation," Journal of Magnetic Resonance, vol. 190, no. 2, pp. 255-270, 2008.

[43] I. Podlubny, Fractional Differential Equations, Academic Press, San Diego, CA, USA, 1999.

[44] G. Zaslavsky, "Chaos, fractional kinetics, and anomalous transport," Physics Reports, vol. 371, no. 6, pp. 461-580, 2002.

[45] C. Li and F. Zeng, "Finite difference methods for fractional differential equations," International Journal of Bifurcation and Chaos in Applied Sciences and Engineering, vol. 22, Article ID 1230014, 2012.

[46] Q. M. Al-Mdallal, H. Yusuf, and A. Ali, "A novel algorithm for time-fractional foam drainage equation," Alexandria Engineering Journal, vol. 59, no. 3, pp. 1607-1612, 2020.

[47] A. Thabet, Q. Al-Mdallal, and F. Jarad, "Fractional logistic models in the frame of fractional operators generated by conformable derivatives," Chaos, Solitons \& Fractals, vol. 119, pp. 94-101, 2019

[48] R. Woodward, F. De Luca, A. Roberts, and A. Bismarck, "High-surface-area, emulsion-templated carbon foams by activation of polyhipes derived from pickering emulsions," Materials, vol. 9, no. 9, p. 776, 2016.

[49] H. P. Ahmad, A. Saeed, A. Masoud, F. M. Boshra, M. Omid, and W. Somchai, "An updated review on application of nanofluids in heat exchangers for saving energy," Energy Conversion and Management, vol. 198, Article ID 111886, 2019.

[50] S. Emad, M. Mohammad, R. Saidur et al., "A comprehensive review on graphene nanofluids: recent research, development and applications," Energy Conversion and Management, vol. 111, pp. 466-487, 2016.

[51] H. Markovitz and B. D. Coleman, Advances in Applied Mechanic, Vol. 8, Academic Press, New York, NY, USA, 1964.

[52] Z. Aparna, M. Michael, S. K. Pabi, and S. Ghosh, "Thermal conductivity of aqueous $\mathrm{Al} 2 \mathrm{O} 3 / \mathrm{Ag}$ hybrid nanofluid at different temperatures and volume concentrations: an experimental investigation and development of new correlation function," Powder Technology, vol. 343, pp. 714-722, 2019.

[53] L. Botong and L. Fawang, "Boundary layer flows of viscoelastic fluids over a non-uniform permeable surface," Computers \& Mathematics with Applications, vol. 79, pp. 2376-2387, 2019.

[54] J. Zhao, L. Zheng, X. Chen, X. Zhang, and F. Liu, "Unsteady marangoni convection heat transfer of fractional Maxwell fluid with Cattaneo heat flux," Applied Mathematical Modelling, vol. 44, pp. 497-507, 2017.

[55] A. Kumar, D. Rajdev, and D. L. Douglass, "Effect of oxide defect structure on the electrical properties of $\mathrm{ZrO} 2$," Journal of the American Ceramic Society, vol. 55, no. 9, pp. 439-445, 1972.

[56] H. Liu, W. Sun, X. Xie et al., "Adjustable thermal expansion properties in $\mathrm{Zr} 2 \mathrm{MoP} 2 \mathrm{O} 12 / \mathrm{ZrO} 2$ ceramic composites," Frontiers in Chemistry, vol. 6, p. 347, 2018.

[57] I. S. Mohamed, C. S. Raj, S. Arul, and P. Rathnakumar, "Heat transfer intensification of zirconia/water nanofluid," International Journal of Advanced Chemistry, vol. 13, pp. 1-8, 2017.

[58] R. W. Vest, N. M. Tallan, and W. C. TRIPP, "Electrical properties and defect structure of Zirconia: I, monoclinic phase," Journal of the American Ceramic Society, vol. 47, no. 12, pp. 635-640, 1964.
[59] H. Xie, B. Jiang, B. Liu, Q. Wang, J. Xu, and F. Pan, "An investigation on the tribological performances of the $\mathrm{SiO} 2 /$ MoS2 hybrid nanofluids for magnesium alloy-steel contacts," Nanoscale Research Letters, vol. 11, no. 1, pp. 329-336, 2016.

[60] I. Pop and D. B. Ingham, Transport Phenomena in Porous Media Ii, Pergamon, Turkey, 2002.

[61] L. Zheng, Y. Liu, and X. Zhang, "A new model for plasticviscoelastic magnetohydrodynamic (MHD) flow with radiation thermal transfer," International Journal of Nonlinear Sciences and Numerical Stimulation, vol. 14, no. 7-8, pp. 435-441, 2013.

[62] F. Liu, P. Zhuang, V. Anh, I. Turner, and K. Burrage, "Stability and convergence of the difference methods for the space-time fractional advection-diffusion equation," Applied Mathematics and Computation, vol. 191, no. 1, pp. 12-20, 2007.

[63] A. Jan, B. Mir, and Ahmed, "Hybrid nanofluids: an overview of their synthesis and thermophysical properties," Applied Physics, 2019.

[64] W. Yu and S. U. S. Choi, "The role of interfacial layers in the enhanced thermal conductivity of nanofluids: a renovated Maxwell model," Journal of Nanoparticle Research, vol. 5, no. $1 / 2$, pp. 167-171, 2003.

[65] R. L. Hamilton and O. K. Crosser, "Thermal conductivity of heterogeneous two-component systems," Industrial \& Engineering Chemistry Fundamentals, vol. 1, no. 3, pp. 187-191, 1962.

[66] P. R. Summer, Heat Transfer Conference. Collocated with the Asme 2005 Pacific Rim, ASME, New York, NY, USA. 